\title{
Selenacalix[4]selenophene: Synthesis, Structure, and Gel Formation of Cyclic Selenoether of Selenophene
}

\author{
Masashi Hasegawa, ${ }^{* \dagger}$ Shiori Haga ${ }^{\dagger}{ }^{T}$ Tohru Nishinaga, ${ }^{\ddagger}$ and Yasuhiro Mazaki ${ }^{\dagger}$ \\ + Graduate School of Science, Kitasato University, 1-15-1 Kitasato, Minami-ku, Sagamihara, \\ Kanagawa 252-0373 \\ masasi.h@kitasato-u.ac.jp \\ *Department of Chemistry, Graduate School of Science, Tokyo Metropolitan University, Hachioji, \\ Tokyo 192-0397, Japan
}

\section{Supporting Information}

S1. General

p. 2

S2. Synthesis of $\mathbf{4}$

p.3

S3. X-ray Analysis

p.6

S4. Electronic Spectra

p.11

S5. Geometry Optimization

p.12

S6. Molecular Orbital

p.19

S7. TD-DFT Calculations

p.20

S8. Cyclic Voltammetry

p. 22

S9. Gelation Properties in Various Solvents

p. 22

S10. SEM Measurements

p. 23

S11. XRD Analysis of Gel and Simulated Powder Pattern of Single Crystal

$\mathrm{S} 12$. Figure $\mathrm{S} 11 .{ }^{1} \mathrm{H}$ and ${ }^{13} \mathrm{C}$ NMR Charts

p. 25

S13. Figure S12. VT-NMR Chart of 4

p. 33

S14. Figure S13. MS Spectrum Charts

p.34

S15. References

p.36 


\section{S1. General}

${ }^{1} \mathrm{H}$ and ${ }^{13} \mathrm{C}$ NMR spectra were recorded on Bruker AVANCE-II 600 (600 MHz for ${ }^{1} \mathrm{H}$ NMR, 150 $\mathrm{MHz}$ for ${ }^{13} \mathrm{C}$ and $114 \mathrm{MHz}$ for ${ }^{77} \mathrm{Se} \mathrm{NMR}$ ) or Bruker AVANCE-II 400 (400 MHz for ${ }^{1} \mathrm{H}$ NMR, 100 $\mathrm{MHz}$ for ${ }^{13} \mathrm{C}$ and $76 \mathrm{MHz}$ for ${ }^{77} \mathrm{Se} \mathrm{NMR}$ ) instruments. Spectra are reported in $\delta$, referenced to internal tetramethylsilane $\left(\mathrm{Me}_{4} \mathrm{Si}\right)$. Mass spectra and high-resolution mass spectra were recorded on Thermo Scientific, Exactive Plus Orbitrap Mass Spectrometer for electrospray ionization (ESI). Melting points were determined with Yanaco melting point apparatus. IR spectra were recorded on JASCO FT/IR610 spectrometer. UV-Vis spectra were recorded on JASCO V-560 spectrometer.

Starting 3,4-dibromoselenophene was prepared according to literature procedure as shown in Scheme S1. ${ }^{1}$ Selenophene (CAS RN. 288-05-1) and 4-tert-Butylphenylboronic Acid (CAS RN. 123324-71-0) were purchased from Tokyo Chemical Industry Co. Ltd, (TCI). Tributyltinselenide, $\left(\mathrm{Bu}_{3} \mathrm{Sn}\right)_{2} \mathrm{Se}$, was prepared according to literature procedure in $50 \mathrm{mmol}$ scale (Scheme S2). ${ }^{2}$ Other chemicals were purchased from FUJIFILM Wako Pure Chemical Corporation. Column chromatography was carried out using Kanto Chemical silica gel 60N, 60-210mm meshes. All solvents were dried by conventional procedures and distilled before use. ${ }^{3}$

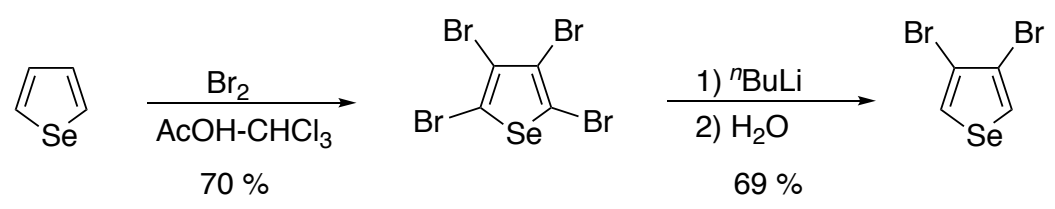

Scheme S1. Synthesis of 3,4-dibromoselenophene from selenophene

$$
\text { Se } \begin{aligned}
& \stackrel{\text { 1) } \mathrm{NaOH}}{\text { 2) } \mathrm{Rongalit}} \\
& \text { 3) } \mathrm{Bu}_{3} \mathrm{SnCl} \\
& 67 \%
\end{aligned}
$$

Scheme S2. Synthesis of $\left.\mathrm{Se}(\mathrm{SnBu})_{2}\right)_{2}$ 


\section{S2. Synthesis of 4}

Synthesis of 3,4-bis(4-t-butylphenyl)selenophene (7)
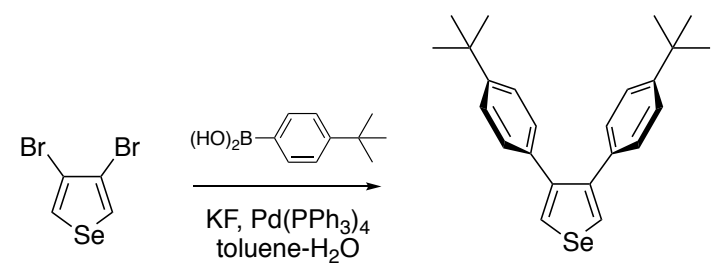

7

A mixture of 3,4-dibromoselenophene (1.01 g, $3.51 \mathrm{mmol})$, 4-t-butylphenylboronic acid (1.78 g, 10.0 $\mathrm{mmol}), \mathrm{KF}(582 \mathrm{mg}, 10.0 \mathrm{mmol})$, and $\mathrm{Pd}\left(\mathrm{PPh}_{3}\right)_{4}(289 \mathrm{mg}, 0.25 \mathrm{mmol})$ in toluene $(20 \mathrm{~mL})$ and $\mathrm{H}_{2} \mathrm{O}$ (20 mmol) was stirred for $14 \mathrm{~h}$ at $130{ }^{\circ} \mathrm{C}$ (oil bath temp.) under Ar. Then, the reaction mixture was filtered through Celite pad. The resultant solution was washed with saturated brine and dried over $\mathrm{Na}_{2} \mathrm{SO}_{4}$. After evaporation, the residue was purified by column chromatography on silica gel with the elution of $\mathrm{CH}_{2} \mathrm{Cl}_{2}$-hexane (v/v = 1:9) to give while solid of 7 (1.0 g, 72\%). Data for 7: M.p. $=164$ $165{ }^{\circ} \mathrm{C}$; HRMS (ESI-orbitrap) calcd for $\mathrm{C}_{24} \mathrm{H}_{27} \mathrm{SeBr}_{2}[\mathrm{M}+\mathrm{H}]^{+}$: 554.9619; found: 554.9620; ${ }^{1} \mathrm{H}$ NMR $\left(400 \mathrm{MHz}, \mathrm{CDCl}_{3}\right) \delta 7.91(\mathrm{~s}, 2 \mathrm{H}), 7.23-7.25(\mathrm{~m}, 4 \mathrm{H}), 7.08-7.10(\mathrm{~m}, 4 \mathrm{H}), 1.31(\mathrm{~s}, 18 \mathrm{H}) ;{ }^{13} \mathrm{C} \mathrm{NMR}$ $\left(100 \mathrm{MHz}, \mathrm{CDCl}_{3}\right) \delta 149.6,144.4,135.3,128.6,128.2,124.8,34.5,31.3 ;{ }^{77} \mathrm{Se} \mathrm{NMR}\left(76 \mathrm{MHz}, \mathrm{CDCl}_{3}\right)$ $\delta$ 576.2; IR (KBr) 2995, 2903, 2865, 1520, 1463, 1362, 1269, 1111, 842, $788 \mathrm{~cm}^{-1}$.

\section{Synthesis of 2,5-dibromo-3,4-bis(4-t-butylphenyl)selenophene (8)}

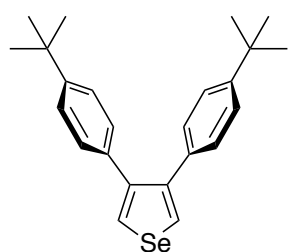

5

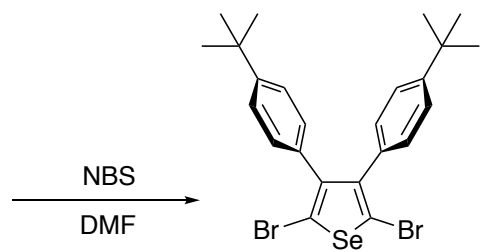

8

To a solution of 7 (300 mg, $0.76 \mathrm{mmol})$ in DMF (1.3 mL), NBS (291 mg, $1.63 \mathrm{mmol})$ in DMF (1.3 $\mathrm{mL}$ ) was added at $0{ }^{\circ} \mathrm{C}$. After stirring for $1 \mathrm{~h}$ at $0{ }^{\circ} \mathrm{C}$, the mixture was further stirred for additional $2 \mathrm{~h}$ at rt. Then, water was added to the reaction mixture. The products were extracted with $\mathrm{CH}_{2} \mathrm{Cl}_{2}$, and the combined organic phase was washed with $\mathrm{NaHCO}_{3}$ and saturated brine. The resultant solution was dried over $\mathrm{MgSO}_{4}$. After evaporation, the residue was purified by column chromatography on silica gel with the elution of $\mathrm{CH}_{2} \mathrm{Cl}_{2}$-hexane ( $\left.\mathrm{v} / \mathrm{v}=1: 10\right)$ to give while solid of $8(269 \mathrm{mg}, 64 \%)$. Data for 8: M.p. $=159-160^{\circ} \mathrm{C}$; HRMS (ESI-orbitrap) calcd for $\mathrm{C}_{24} \mathrm{H}_{29} \mathrm{Se}[\mathrm{M}+\mathrm{H}]^{+}$: 397.1429; found: 397.1428; ${ }^{1} \mathrm{H}$ NMR (400 MHz, $\left.\mathrm{CDCl}_{3}\right) \delta$ 7.17-7.19 (m, 4H); 6.90-6.92 (m, 4H); 1.25 (s, 18H); ${ }^{13} \mathrm{C}$ NMR $(100$ $\left.\mathrm{MHz}, \mathrm{CDCl}_{3}\right) \delta 150.2,145.2,133.1,129.8,124.5,112.0,34.5,31.2 ;{ }^{77} \mathrm{Se} \mathrm{NMR}\left(76 \mathrm{MHz}, \mathrm{CDCl}_{3}\right)$ $\delta$ 715.8; IR (KBr) 2958, 2898, 2863, 1496, 1463, 1363, 1266, 1121, 1019, 973, $836 \mathrm{~cm}^{-1}$. 

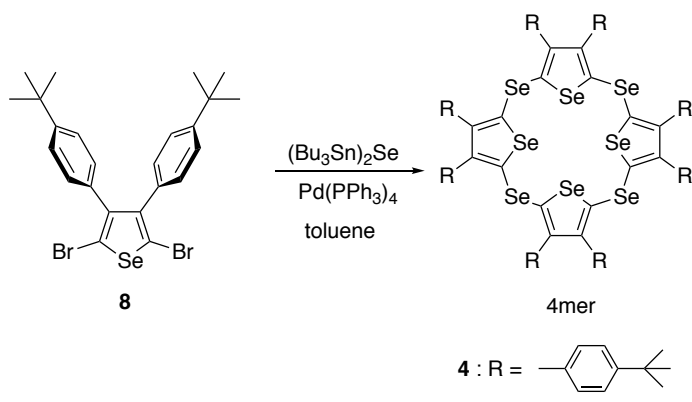

$52 \%$

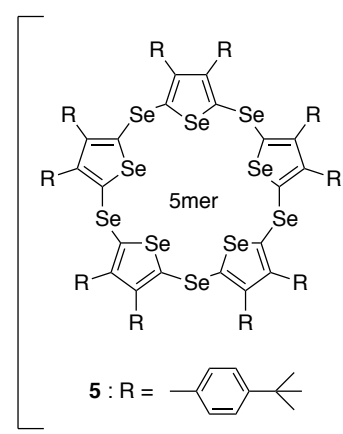

$15 \%$

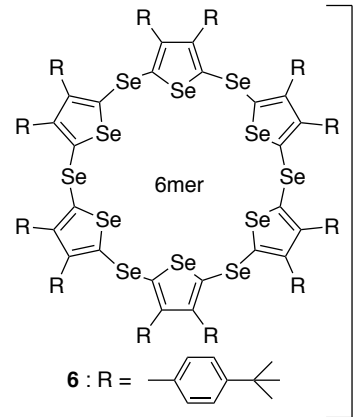

unseparable

A mixture of $8(227 \mathrm{mg}, 0.41 \mathrm{mmol}),\left(\mathrm{Bu} \mathrm{S}_{3} \mathrm{Sn}\right)_{2} \mathrm{Se}(270 \mathrm{mg}, 0.41 \mathrm{mmol})$, and $\mathrm{Pd}\left(\mathrm{PPh}_{3}\right)_{4}(54 \mathrm{mg}, 0.047$ $\mathrm{mmol}$ ) in degassed toluene $\left(10 \mathrm{~mL}, 41 \mathrm{mM}\right.$ ) was stirred at $120^{\circ} \mathrm{C}$ (oil bath temp.) under Ar for 3 days. Then, the solvent was removed, and the residue was soluble in $\mathrm{CH}_{2} \mathrm{Cl}_{2}(30 \mathrm{~mL})$. A saturated aqueous KF solution was added to the solution, and stirred for $1 \mathrm{~h}$. The solution was filtrated through Celite pad. The resultant organic layer was separated, washed with saturated brine, and dried over $\mathrm{Na}_{2} \mathrm{SO}_{4}$. After evaporation, the residue was purified on silica gel containing $10 \mathrm{wt} \% \mathrm{~K}_{2} \mathrm{CO}_{3}$ with elution of $\mathrm{CH}_{2} \mathrm{Cl}_{2}$-hexane ( $\mathrm{v} / \mathrm{v}=1$ ) solution (Figure $\mathrm{S} 11 \mathrm{~g}$ ). Recrystallization from $\mathrm{CH}_{2} \mathrm{Cl}_{2}$-hexane at $-20^{\circ} \mathrm{C}$ gave pale yellow powder of $\mathbf{4}(49 \mathrm{mg})$. Further purification of the filtrate was carried with GPC to give three fractions of 4 (44 mg) and a mixture containing 4 and 5, and a mixture containing 4-6 (Figure S11h). The yield was calculated by adding the isolated $\mathbf{4}$ and the yields determined by NMR spectra; 4: 52\%, 5: 15\%; 6: 5\%. In total, compound 4 was isolated in $92 \mathrm{mg}(48 \%)$.

Data for 4: pale yellow powder; M.p. $=386-387{ }^{\circ} \mathrm{C}$; HRMS (ESI-orbitrap) calcd for $\mathrm{C}_{96} \mathrm{H}_{105} \mathrm{Se}_{8}$ $[\mathrm{M}+\mathrm{H}]^{+}: m / z=1890.1609$, Found 1890.1609; ${ }^{1} \mathrm{H} \mathrm{NMR}\left(400 \mathrm{MHz}, \mathrm{CDCl}_{3}\right) \delta 7.12-7.14(\mathrm{~m}, 16 \mathrm{H})$, 6.87-6.89 (m, 16H), $1.24(\mathrm{~s}, 72 \mathrm{H}) ;{ }^{13} \mathrm{C}$ NMR $\left(100 \mathrm{MHz}, \mathrm{CDCl}_{3}\right) \delta 149.8,148.1,134.5,133.3,129.6$, 124.4, 34.4, 31.3; ${ }^{77} \mathrm{Se} \mathrm{NMR}\left(76 \mathrm{MHz}, \mathrm{CDCl}_{3}\right) \delta$ 715.4, 338.2; IR (KBr) 2962, 2904, 2866, 1520 , $1474,1362,1269,1121,1019,838 \mathrm{~cm}^{-1}$.

Compound $\mathbf{5}$ and $\mathbf{6}$ are not isolated as pure form, and hence the following data are corrected in a mixture of 4, 5, and 6; Data for 5: HRMS (ESI-orbitrap) calcd for $\mathrm{C}_{120} \mathrm{H}_{130} \mathrm{Se}_{10} \mathrm{~K}[\mathrm{M}+\mathrm{K}]^{+}: m / z=$ 2402.1542, Found 2402.1545; ${ }^{1} \mathrm{H}$ NMR (400 MHz, $\left.\mathrm{CDCl}_{3}\right) \delta$ 7.07-7.09 (m, 20H), 6.82-6.85 (m, 20H), 1.22 (s, 90H); ${ }^{13} \mathrm{C}$ NMR (100 MHz, $\left.\mathrm{CDCl}_{3}\right) \delta 149.6,148.5,134.5,133.0,129.8,124.3,34.4,31.3$; ${ }^{77} \mathrm{Se}$ NMR (76 MHz, $\mathrm{CDCl}_{3}$ ) 722.9, 453.7. Data for 6: HRMS (ESI-orbitrap) calcd for $\mathrm{C}_{144} \mathrm{H}_{156} \mathrm{~S}_{12} \mathrm{~K}$ $[\mathrm{M}+\mathrm{K}]^{+}: m / z=2873.1941$, Found 2873.1943; ${ }^{1} \mathrm{H} \mathrm{NMR}\left(400 \mathrm{MHz}, \mathrm{CDCl}_{3}\right) \delta 7.03-7.05(\mathrm{~m}, 24 \mathrm{H})$, $6.77-6.79(\mathrm{~m}, 24 \mathrm{H})$. 


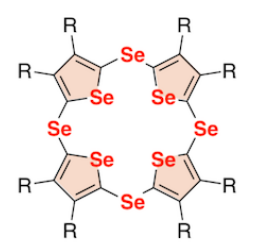

4

reductive elimination
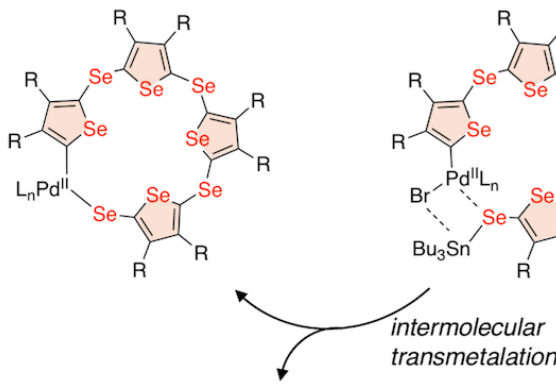

$\mathrm{Bu}_{3} \mathrm{SnBr}$
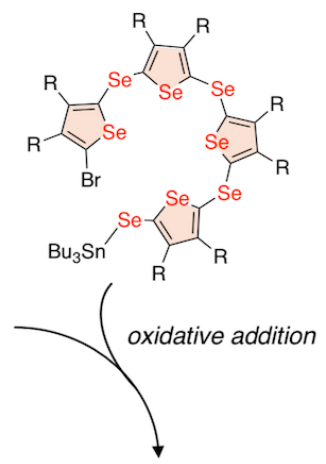

$\mathrm{L}: \mathrm{PPh}_{3}$

R: -

Scheme S3. Possible reaction mechanism for the synthesis of 4 


\section{S3. X-ray Analysis}

Single crystals suitable for X-ray analysis were obtained from recrystallization from $\mathrm{CH}_{2} \mathrm{Cl}_{2}$-hexane. The intensity data were collected on Rigaku XtaLAB Synergy-R/DW using monochromated Cu-K $\alpha$ radiation $(\lambda=1.54184 \AA)$. A colorless tin needle with the dimensions of $0.02 \times 0.02 \times 0.12 \mathrm{~mm}^{3}$ was chosen for the analysis. The crystal structure was solved by the direct method (SHELEXT) and refined by full-matrix least-squares method by using Olex2. Three $t$-butyl groups were disordered, and some carbon atoms are treated as SIMU, RIGU, or ISOR. All hydrogen atoms were refined with isotropic displacement parameters.

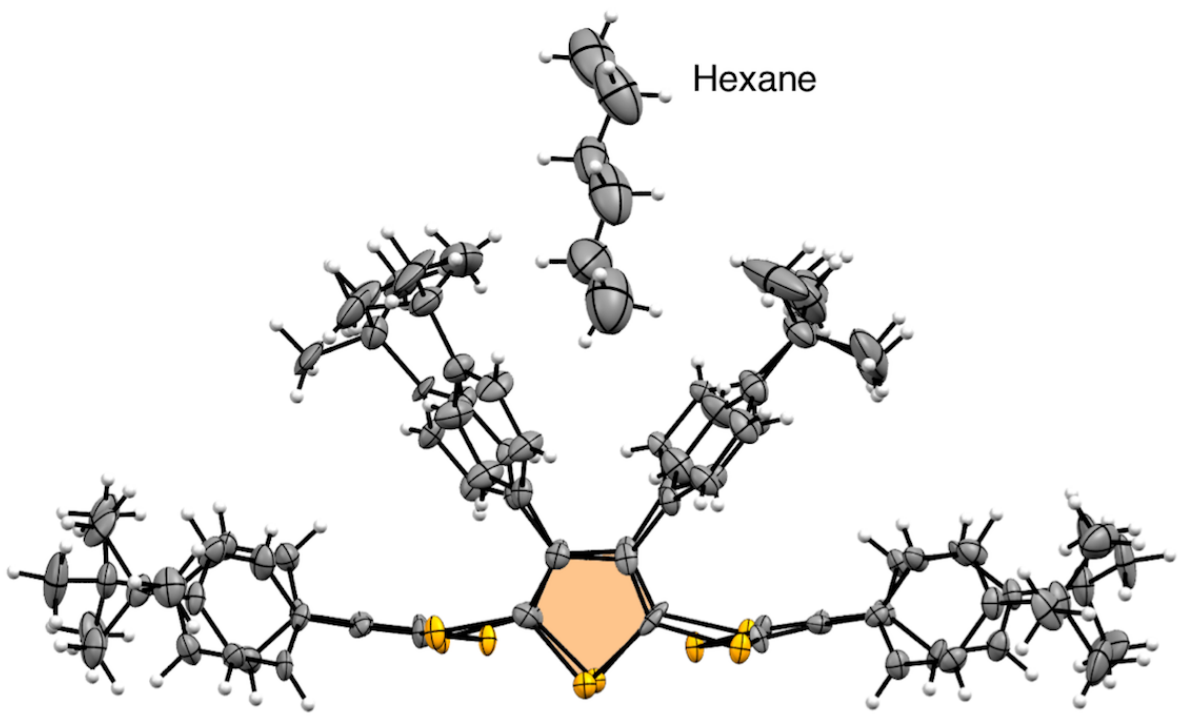

Figure S1. ORTEP drawing of 4 (side view)

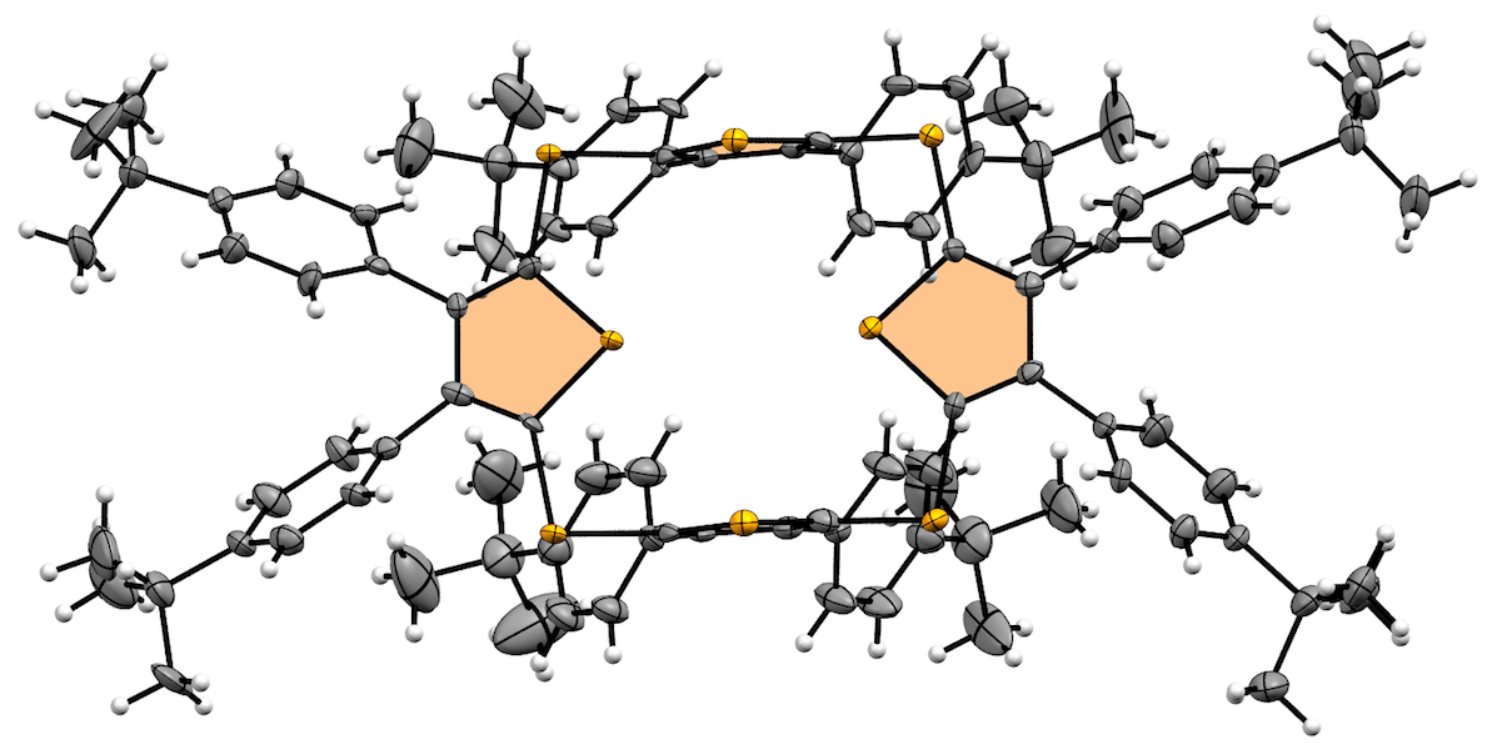

Figure S2. ORTEP drawing of 4 (bottoms view) 


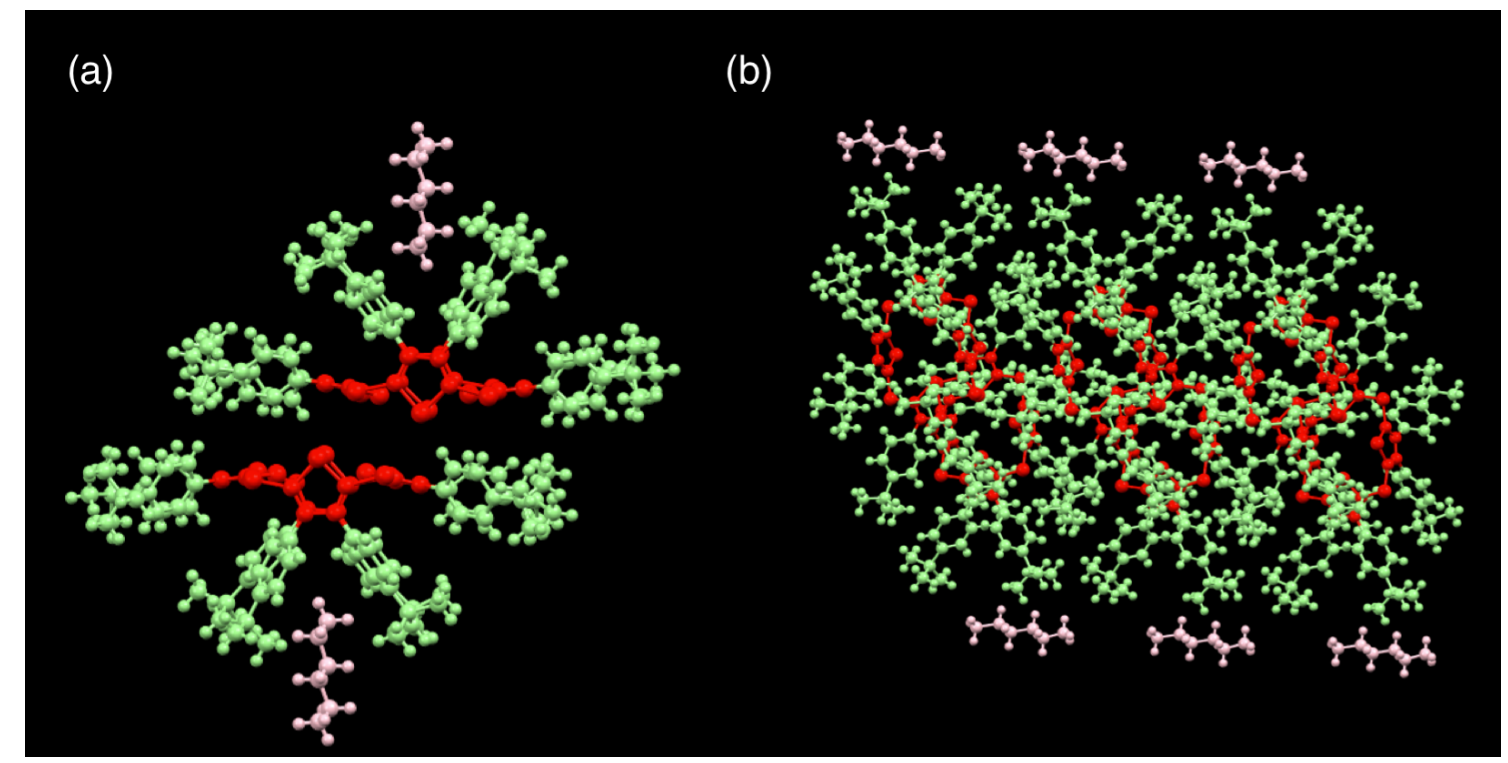

Figure S3. Packing diagram of 4 


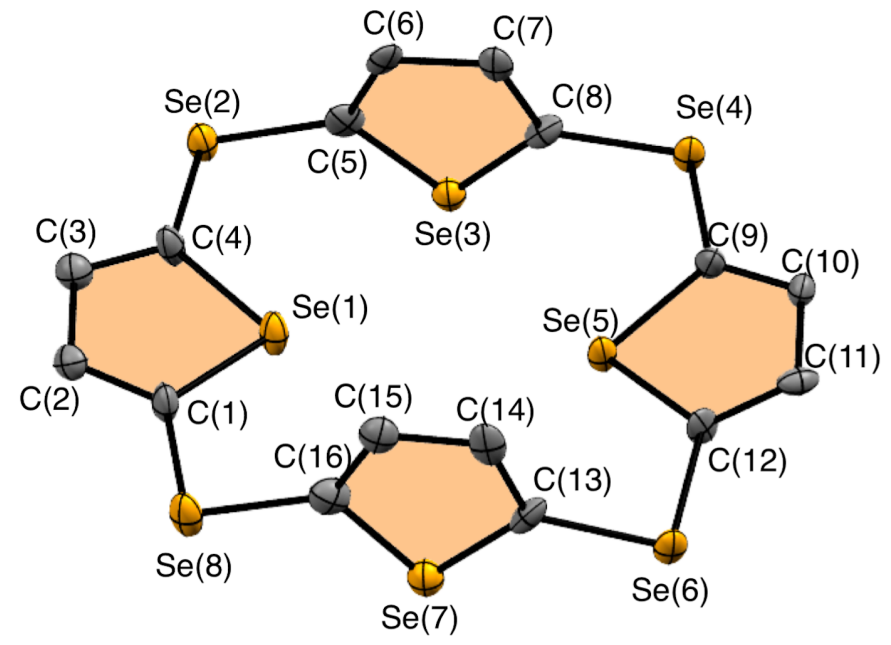

Figure S4. ORTEP drawing of the core of 4. t-butylphenyl groups are omitted for clarity.

Table S1. Selected bond length (A) of the cyclic core

\begin{tabular}{|c|c|c|c|c|c|}
\hline Atom & Atom & Length $(\AA)$ & Atom & Atom & Length $(\AA)$ \\
\hline $\operatorname{Se}(1)$ & $C(1)$ & $1.871(6)$ & $\mathrm{C}(1)$ & $C(2)$ & $1.350(9)$ \\
\hline $\operatorname{Se}(1)$ & $C(4)$ & $1.878(6)$ & $\mathrm{C}(2)$ & $C(3)$ & $1.458(7)$ \\
\hline $\operatorname{Se}(2)$ & $C(5)$ & $1.898(6)$ & $\mathrm{C}(3)$ & $C(4)$ & $1.354(9)$ \\
\hline $\operatorname{Se}(2)$ & $C(4)$ & $1.906(5)$ & $\mathrm{C}(5)$ & $C(6)$ & $1.367(9)$ \\
\hline $\operatorname{Se}(3)$ & $C(5)$ & $1.876(6)$ & $\mathrm{C}(6)$ & $C(7)$ & $1.441(9)$ \\
\hline $\operatorname{Se}(3)$ & $C(8)$ & $1.861(6)$ & $C(7)$ & $\mathrm{C}(8)$ & $1.397(8)$ \\
\hline $\operatorname{Se}(4)$ & $C(8)$ & $1.909(6)$ & $\mathrm{C}(9)$ & $C(10)$ & $1.340(8)$ \\
\hline $\operatorname{Se}(4)$ & $\mathrm{C}(9)$ & $1.910(5)$ & $C(10)$ & $C(11)$ & $1.457(7)$ \\
\hline $\operatorname{Se}(5)$ & $\mathrm{C}(9)$ & $1.875(6)$ & $\mathrm{C}(11)$ & $\mathrm{C}(12)$ & $1.357(8)$ \\
\hline $\operatorname{Se}(5)$ & $C(12)$ & $1.879(5)$ & $C(13)$ & $C(14)$ & $1.384(9)$ \\
\hline $\operatorname{Se}(6)$ & $C(12)$ & $1.898(5)$ & $C(14)$ & $C(15)$ & $1.403(9)$ \\
\hline $\operatorname{Se}(6)$ & $C(13)$ & $1.897(6)$ & $C(15)$ & $C(16)$ & $1.381(9)$ \\
\hline $\operatorname{Se}(7)$ & $C(13)$ & $1.861(7)$ & & & \\
\hline $\operatorname{Se}(7)$ & $C(16)$ & $1.877(6)$ & & & \\
\hline $\operatorname{Se}(8)$ & $C(1)$ & $1.905(5)$ & & & \\
\hline $\operatorname{Se}(8)$ & $C(16)$ & $1.882(7)$ & & & \\
\hline
\end{tabular}


Table S2. Selected bond angles (A) of the cyclic core

\begin{tabular}{cccccccc}
\hline Atom & Atom & Atom & Length $(\AA)$ & Atom & Atom & Atom & Length $(\AA)$ \\
\hline $\operatorname{Se}(1)$ & $\mathrm{C}(1)$ & $\mathrm{Se}(8)$ & $123.4(3)$ & $\mathrm{Se}(8)$ & $\mathrm{C}(1)$ & $\mathrm{C}(2)$ & $123.4(4)$ \\
$\mathrm{Se}(1)$ & $\mathrm{C}(4)$ & $\mathrm{Se}(2)$ & $121.7(3)$ & $\mathrm{Se}(8)$ & $\mathrm{C}(16)$ & $\mathrm{C}(15)$ & $129.0(5)$ \\
$\mathrm{Se}(1)$ & $\mathrm{C}(4)$ & $\mathrm{C}(3)$ & $112.4(4)$ & $\mathrm{C}(1)$ & $\mathrm{Se}(1)$ & $\mathrm{C}(4)$ & $86.2(3)$ \\
$\mathrm{Se}(1)$ & $\mathrm{C}(1)$ & $\mathrm{C}(2)$ & $113.2(4)$ & $\mathrm{C}(1)$ & $\mathrm{C}(2)$ & $\mathrm{C}(3)$ & $113.7(5)$ \\
$\mathrm{Se}(2)$ & $\mathrm{C}(5)$ & $\mathrm{Se}(3)$ & $120.4(3)$ & $\mathrm{C}(1)$ & $\mathrm{Se}(8)$ & $\mathrm{C}(16)$ & $98.3(2)$ \\
$\operatorname{Se}(2)$ & $\mathrm{C}(4)$ & $\mathrm{C}(3)$ & $125.6(4)$ & $\mathrm{C}(2)$ & $\mathrm{C}(3)$ & $\mathrm{C}(4)$ & $114.5(5)$ \\
$\operatorname{Se}(2)$ & $\mathrm{C}(5)$ & $\mathrm{C}(6)$ & $127.5(5)$ & $\mathrm{C}(4)$ & $\mathrm{Se}(2)$ & $\mathrm{C}(5)$ & $98.9(2)$ \\
$\operatorname{Se}(3)$ & $\mathrm{C}(5)$ & $\mathrm{C}(6)$ & $111.9(5)$ & $\mathrm{C}(5)$ & $\mathrm{C}(6)$ & $\mathrm{C}(7)$ & $115.5(5)$ \\
$\operatorname{Se}(3)$ & $\mathrm{C}(8)$ & $\mathrm{C}(7)$ & $112.5(4)$ & $\mathrm{C}(5)$ & $\mathrm{Se}(3)$ & $\mathrm{C}(8)$ & $87.1(3)$ \\
$\operatorname{Se}(3)$ & $\mathrm{C}(8)$ & $\mathrm{Se}(4)$ & $119.2(3)$ & $\mathrm{C}(6)$ & $\mathrm{C}(7)$ & $\mathrm{C}(8)$ & $113.0(5)$ \\
$\operatorname{Se}(4)$ & $\mathrm{C}(9)$ & $\mathrm{C}(10)$ & $125.9(4)$ & $\mathrm{C}(8)$ & $\mathrm{Se}(4)$ & $\mathrm{C}(9)$ & $99.2(2)$ \\
$\operatorname{Se}(4)$ & $\mathrm{C}(9)$ & $\mathrm{Se}(5)$ & $121.0(3)$ & $\mathrm{C}(9)$ & $\mathrm{Se}(5)$ & $\mathrm{C}(12)$ & $86.2(2)$ \\
$\operatorname{Se}(4)$ & $\mathrm{C}(8)$ & $\mathrm{C}(7)$ & $128.0(5)$ & $\mathrm{C}(9)$ & $\mathrm{C}(10)$ & $\mathrm{C}(11)$ & $114.8(5)$ \\
$\operatorname{Se}(5)$ & $\mathrm{C}(12)$ & $\mathrm{Se}(6)$ & $122.7(3)$ & $\mathrm{C}(10)$ & $\mathrm{C}(11)$ & $\mathrm{C}(12)$ & $113.7(5)$ \\
$\operatorname{Se}(5)$ & $\mathrm{C}(9)$ & $\mathrm{C}(10)$ & $112.8(4)$ & $\mathrm{C}(12)$ & $\mathrm{Se}(6)$ & $\mathrm{C} 13)$ & $97.2(2)$ \\
$\operatorname{Se}(5)$ & $\mathrm{C}(12)$ & $\mathrm{C}(11)$ & $112.6(4)$ & $\mathrm{C}(13)$ & $\mathrm{C}(14)$ & $\mathrm{C}(15)$ & $114.3(6)$ \\
$\operatorname{Se}(6)$ & $\mathrm{C}(12)$ & $\mathrm{C}(11)$ & $124.7(4)$ & $\mathrm{C}(13)$ & $\mathrm{Se}(7)$ & $\mathrm{C}(16)$ & $86.8(3)$ \\
$\operatorname{Se}(6)$ & $\mathrm{C}(13)$ & $\mathrm{C}(14)$ & $127.8(5)$ & $\mathrm{C}(14)$ & $\mathrm{C}(15)$ & $\mathrm{C}(16)$ & $115.6(6)$ \\
$\operatorname{Se}(6)$ & $\mathrm{C}(13)$ & $\mathrm{Se}(7)$ & $119.9(3)$ & & & & \\
$\operatorname{Se}(7)$ & $\mathrm{C}(16)$ & $\mathrm{Se}(8)$ & $119.7(3)$ & & & & \\
$\operatorname{Se}(7)$ & $\mathrm{C}(16)$ & $\mathrm{C}(15)$ & $111.1(5)$ & & & & \\
$\operatorname{Se}(7)$ & $\mathrm{C}(13)$ & $\mathrm{C}(14)$ & $112.1(5)$ & & & & \\
\hline
\end{tabular}


Table S3. X-ray analyses of 4

\begin{tabular}{|c|c|}
\hline Identification code & 4 \\
\hline Crystal description & Colorless \\
\hline Empirical formula & $\mathrm{C}_{102} \mathrm{H}_{118} \mathrm{Se}_{8}$ \\
\hline Moiety formula & $\mathrm{C}_{96} \mathrm{H}_{104} \mathrm{Se}_{8} \cdot{ }^{\circ} \mathrm{C}_{6} \mathrm{H}_{14}$ \\
\hline Formula weight & 1975.64 \\
\hline Temperature & $120 \mathrm{~K}$ \\
\hline Wavelength & $\mathrm{Cu}-K \alpha 1.54184 \AA$ \\
\hline Crystal system & Orthorhombic \\
\hline Space group & $P 2_{1} / \mathrm{c}(\# 14)$ \\
\hline \multirow[t]{6}{*}{ Unite cell dimensions } & $a=12.2822(1) \AA$ \\
\hline & $b=36.6384(5) \AA$ \\
\hline & $c=21.9106(3) \AA$ \\
\hline & $\alpha=90^{\circ}$ \\
\hline & $\beta=104.297(1)^{\circ}$ \\
\hline & $\gamma=90^{\circ}$ \\
\hline Volume & $2913.0(2) \AA^{3}$ \\
\hline$Z$ & 4 \\
\hline Density (calc.) & $1.373 \mathrm{gcm}^{-1}$ \\
\hline$\mu / \mathrm{mm}^{-1}$ & 3.904 \\
\hline $\mathrm{F}(000)$ & 4008 \\
\hline Crystal size & $0.02 \times 0.02 \times 0.12 \mathrm{~cm}^{3}$ \\
\hline \multirow[t]{3}{*}{ Index ranges } & $-14<=\mathrm{h}<=14$ \\
\hline & $-42 \leq \mathrm{k} \leq 29$ \\
\hline & $-25 \leq 1 \leq 26$ \\
\hline Reflections collected & 43354 \\
\hline Independent & $16039\left[\mathrm{R}_{\mathrm{int}}=0.0771\right.$, \\
\hline reflections & $\left.\mathrm{R}_{\text {sigma }}=0.0750\right]$ \\
\hline $\begin{array}{c}\text { Data/restraints/param } \\
\text { eters }\end{array}$ & $16039 / 201 / 1110$ \\
\hline Goodness-of-fit on $\mathrm{F}^{2}$ & 1.050 \\
\hline Final $R$ indices & $R_{1}=0.0716$ \\
\hline$[I>2 \sigma(I)]$ & $w R_{2}=0.1964$ \\
\hline $\begin{array}{l}\text { Final } R \text { indices } \\
\text { [all data] }\end{array}$ & $\begin{array}{l}R_{1}=0.0852, \\
w R_{2}=0.2205\end{array}$ \\
\hline $\begin{array}{l}\text { CCDC deposition } \\
\text { No. }\end{array}$ & 1974178 \\
\hline
\end{tabular}


S4. Electronic Spectra

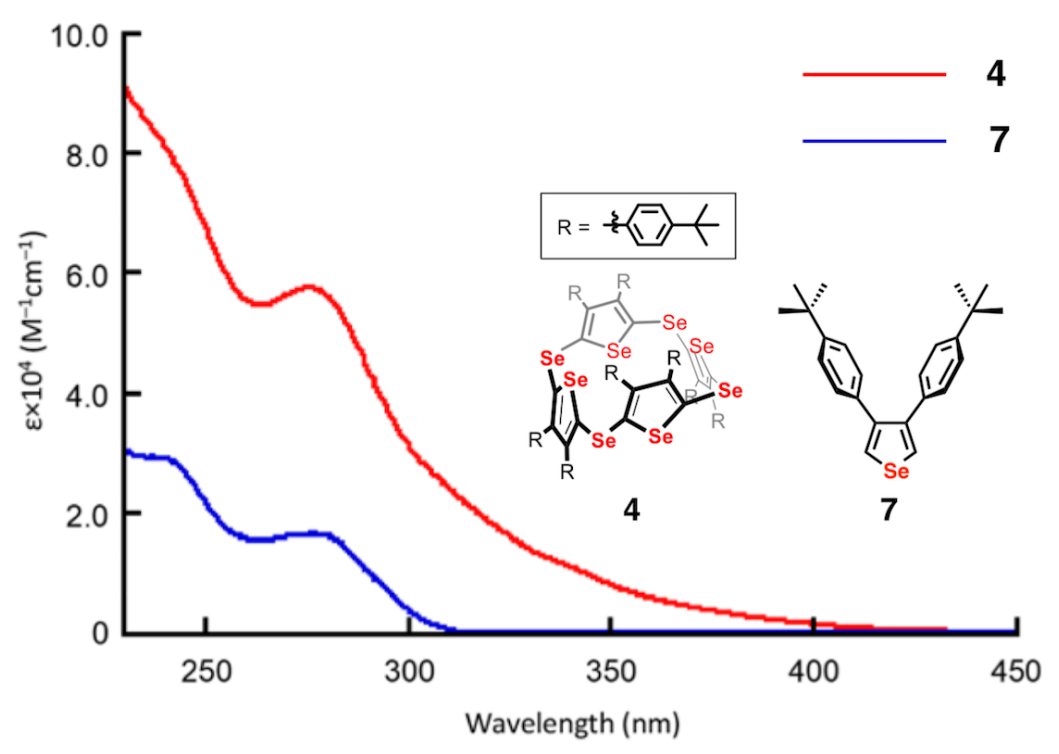

Figure S5. Electronic spectra of $4\left(c=1.2 \times 10^{-5} \mathrm{M}\right)$ and $7\left(\mathrm{c}=1.0 \times 10^{-5} \mathrm{M}\right)$ in $\mathrm{CH}_{2} \mathrm{Cl}_{2}$. 


\section{S5. Geometry Optimization}

The geometry optimization of 4 was performed by DFT calculation (Gaussian 16, Revision A.03) at B3LYP/6-31G(d) level of theory. The minimum energy of each conformation was confirmed by frequency calculations. We started with four conformers of 1,3-alternate, 1,2-alternate, partial cone, and all cone, which are compared as calix[4]arene conformers. Finally, we obtained five conformers having local minimum energy (Figure S4). The alternate conformation with $S_{4}$ symmetry (4-i) was found as the most stable geometry. Another conformation of 4-ii is close to the result of X-ray crystal structure analysis. The atomic coordinates of 4-i and 4-ii were listed below (Table S4).

Table S4. Geometry and total energy of each conformer in 4

\begin{tabular}{|c|c|c|}
\hline $\begin{array}{c}\text { In code } \\
\text { conformation }\end{array}$ & 4-i & all cone \\
\hline Geometry & 1,3 -alternate \\
\hline $\begin{array}{c}\text { Point Group } \\
\text { \# of img }\end{array}$ & $0.00 \mathrm{~kJ} / \mathrm{mol}$ & 0 \\
\hline $\begin{array}{c}\text { Energy in } \mathrm{HF} \\
\text { Relative }\end{array}$ & -22916.0308676 & -22916.0115541 \\
\hline
\end{tabular}

\begin{tabular}{|c|c|c|c|}
\hline ID code & 4-iii & 4-iv & $4-\mathrm{v}$ \\
\hline $\begin{array}{c}\text { Initial } \\
\text { conformation }\end{array}$ & 1,3-alternate & 1,2-alternate & partial cone $(\mathrm{PG})$ \\
\hline Geometry & & & \\
\hline Point Group & $C_{1}$ & $C_{1}$ & $C_{1}$ \\
\hline \# of img & 0 & 0 & 0 \\
\hline Energy in HF & -22916.025804 & -22916.013075 & -22916.0130751 \\
\hline $\begin{array}{c}\text { Relative } \\
\text { energy }\end{array}$ & $+13.9 \mathrm{~kJ} / \mathrm{mol}$ & $+46.7 \mathrm{~kJ} / \mathrm{mol}$ & $+46.7 \mathrm{~kJ} / \mathrm{mol}$ \\
\hline
\end{tabular}


Table S4. Atomic coordinate of optimized structure of 4-i

\begin{tabular}{|c|c|c|c|c|c|}
\hline \multirow{2}{*}{$\begin{array}{l}\text { Center } \\
\text { Number }\end{array}$} & \multirow{2}{*}{$\begin{array}{l}\text { Atomic } \\
\text { Number }\end{array}$} & \multirow{2}{*}{$\begin{array}{l}\text { Atomic } \\
\text { Type }\end{array}$} & \multicolumn{3}{|c|}{ Coordinates (Angstroms) } \\
\hline & & & $X$ & Y & $\mathrm{Z}$ \\
\hline 1 & 6 & 0 & 2.672292 & -1.599284 & 0.949996 \\
\hline 2 & 6 & 0 & 2.456049 & -2.849290 & 1.492205 \\
\hline 3 & 6 & 0 & 1.562154 & -3.680844 & 0.705178 \\
\hline 4 & 6 & 0 & 1.129245 & -3.079748 & -0.457400 \\
\hline 5 & 6 & 0 & -1.599284 & -2.672292 & -0.949996 \\
\hline 6 & 6 & 0 & -2.849290 & -2.456049 & -1.492205 \\
\hline 7 & 6 & 0 & -3.680844 & -1.562154 & -0.705178 \\
\hline 8 & 6 & 0 & -3.079748 & -1.129245 & 0.457400 \\
\hline 9 & 6 & 0 & -2.672292 & 1.599284 & 0.949996 \\
\hline 10 & 6 & 0 & -2.456049 & 2.849290 & 1.492205 \\
\hline 11 & 6 & 0 & -1.562154 & 3.680844 & 0.705178 \\
\hline 12 & 6 & 0 & -1.129245 & 3.079748 & -0.457400 \\
\hline 13 & 6 & 0 & 1.599284 & 2.672292 & -0.949996 \\
\hline 14 & 6 & 0 & 2.849290 & 2.456049 & -1.492205 \\
\hline 15 & 6 & 0 & 3.680844 & 1.562154 & -0.705178 \\
\hline 16 & 6 & 0 & 3.079748 & 1.129245 & 0.457400 \\
\hline 17 & 6 & 0 & -3.250575 & -3.064637 & -2.786070 \\
\hline 18 & 6 & 0 & -3.064637 & -4.435321 & -3.013506 \\
\hline 19 & 6 & 0 & -3.795887 & -2.300769 & -3.831234 \\
\hline 20 & 6 & 0 & -3.402976 & -5.019343 & -4.236099 \\
\hline 21 & 1 & 0 & -2.647834 & -5.053754 & -2.222461 \\
\hline 22 & 6 & 0 & -4.133776 & -2.890346 & -5.043909 \\
\hline 23 & 1 & 0 & -3.948404 & -1.235154 & -3.692741 \\
\hline 24 & 6 & 0 & -3.948351 & -4.264381 & -5.281511 \\
\hline 25 & 1 & 0 & -3.238841 & -6.084406 & -4.357142 \\
\hline 26 & 1 & 0 & -4.547533 & -2.258704 & -5.825311 \\
\hline 27 & 6 & 0 & 3.250575 & 3.064637 & -2.786070 \\
\hline 28 & 6 & 0 & 3.795887 & 2.300769 & -3.831234 \\
\hline 29 & 6 & 0 & 3.064637 & 4.435321 & -3.013506 \\
\hline 30 & 6 & 0 & 4.133776 & 2.890346 & -5.043909 \\
\hline 31 & 1 & 0 & 3.948404 & 1.235154 & -3.692741 \\
\hline 32 & 6 & 0 & 3.402976 & 5.019343 & -4.236099 \\
\hline 33 & 1 & 0 & 2.647834 & 5.053754 & -2.222461 \\
\hline 34 & 6 & 0 & 3.948351 & 4.264381 & -5.281511 \\
\hline 35 & 1 & 0 & 4.547533 & 2.258704 & -5.825311 \\
\hline 36 & 1 & 0 & 3.238841 & 6.084406 & -4.357142 \\
\hline 37 & 6 & 0 & -5.056464 & -1.148481 & -1.092054 \\
\hline 38 & 6 & 0 & -5.386923 & 0.202094 & -1.264427 \\
\hline 39 & 6 & 0 & -6.071133 & -2.101362 & -1.266071 \\
\hline 40 & 6 & 0 & -6.687479 & 0.581568 & -1.599880 \\
\hline 41 & 1 & 0 & -4.615183 & 0.960032 & -1.174066 \\
\hline 42 & 6 & 0 & -7.366205 & -1.712420 & -1.592244 \\
\hline 43 & 1 & 0 & -5.842242 & -3.155709 & -1.139652 \\
\hline 44 & 6 & 0 & -7.710696 & -0.360691 & -1.766509 \\
\hline 45 & 1 & 0 & -6.890510 & 1.638546 & -1.732511 \\
\hline 46 & 1 & 0 & -8.122396 & -2.483516 & -1.711928 \\
\hline 47 & 6 & 0 & 5.056464 & 1.148481 & -1.092054 \\
\hline 48 & 6 & 0 & 5.386923 & -0.202094 & -1.264427 \\
\hline 49 & 6 & 0 & 6.071133 & 2.101362 & -1.266071 \\
\hline 50 & 6 & 0 & 6.687479 & -0.581568 & -1.599880 \\
\hline 51 & 1 & 0 & 4.615183 & -0.960032 & -1.174066 \\
\hline 52 & 6 & 0 & 7.366205 & 1.712420 & -1.592244 \\
\hline 53 & 1 & 0 & 5.842242 & 3.155709 & -1.139652 \\
\hline 54 & 6 & 0 & 7.710696 & 0.360691 & -1.766509 \\
\hline 55 & 1 & 0 & 6.890510 & -1.638546 & -1.732511 \\
\hline 56 & 1 & 0 & 8.122396 & 2.483516 & -1.711928 \\
\hline 57 & 6 & 0 & -3.064637 & 3.250575 & 2.786070 \\
\hline 58 & 6 & 0 & -2.300769 & 3.795887 & 3.831234 \\
\hline 59 & 6 & 0 & -4.435321 & 3.064637 & 3.013506 \\
\hline
\end{tabular}




\begin{tabular}{|c|c|c|c|c|c|}
\hline 60 & 6 & 0 & -2.890346 & 4.133776 & 5.043909 \\
\hline 61 & 1 & 0 & -1.235154 & 3.948404 & 3.692741 \\
\hline 62 & 6 & 0 & -5.019343 & 3.402976 & 4.236099 \\
\hline 63 & 1 & 0 & -5.053754 & 2.647834 & 2.222461 \\
\hline 64 & 6 & 0 & -4.264381 & 3.948351 & 5.281511 \\
\hline 65 & 1 & 0 & -2.258704 & 4.547533 & 5.825311 \\
\hline 66 & 1 & 0 & -6.084406 & 3.238841 & 4.357142 \\
\hline 67 & 6 & 0 & 3.064637 & -3.250575 & 2.786070 \\
\hline 68 & 6 & 0 & 2.300769 & -3.795887 & 3.831234 \\
\hline 69 & 6 & 0 & 4.435321 & -3.064637 & 3.013506 \\
\hline 70 & 6 & 0 & 2.890346 & -4.133776 & 5.043909 \\
\hline 71 & 1 & 0 & 1.235154 & -3.948404 & 3.692741 \\
\hline 72 & 6 & 0 & 5.019343 & -3.402976 & 4.236099 \\
\hline 73 & 1 & 0 & 5.053754 & -2.647834 & 2.222461 \\
\hline 74 & 6 & 0 & 4.264381 & -3.948351 & 5.28151 \\
\hline 75 & 1 & 0 & 2.258704 & -4.547533 & 5.82531 \\
\hline 76 & 1 & 0 & 6.084406 & -3.238841 & 4.357142 \\
\hline 77 & 6 & 0 & 1.148481 & -5.056464 & 1.092054 \\
\hline 78 & 6 & 0 & -0.202094 & -5.386923 & 1.264427 \\
\hline 79 & 6 & 0 & 2.101362 & -6.071133 & 1.266071 \\
\hline 80 & 6 & 0 & -0.581568 & -6.687479 & 1.599880 \\
\hline 81 & 1 & 0 & -0.960032 & -4.615183 & 1.174066 \\
\hline 82 & 6 & 0 & 1.712420 & -7.366205 & 1.592244 \\
\hline 83 & 1 & 0 & 3.155709 & -5.842242 & 1.139652 \\
\hline 84 & 6 & 0 & 0.360691 & -7.710696 & 1.766509 \\
\hline 85 & 1 & 0 & -1.638546 & -6.890510 & 1.732511 \\
\hline 86 & 1 & 0 & 2.483516 & -8.122396 & 1.711928 \\
\hline 87 & 6 & 0 & -1.148481 & 5.056464 & 1.092054 \\
\hline 88 & 6 & 0 & 0.202094 & 5.386923 & 1.264427 \\
\hline 89 & 6 & 0 & -2.101362 & 6.071133 & 1.266071 \\
\hline 90 & 6 & 0 & 0.581568 & 6.687479 & 1.59988 \\
\hline 91 & 1 & 0 & 0.960032 & 4.615183 & 1.17406 \\
\hline 92 & 6 & 0 & -1.712420 & 7.366205 & 1.592244 \\
\hline 93 & 1 & 0 & -3.155709 & 5.842242 & 1.13965 \\
\hline 94 & 6 & 0 & -0.360691 & 7.710696 & 1.76650 \\
\hline 95 & 1 & 0 & 1.638546 & 6.890510 & 1.73251 \\
\hline 96 & 1 & 0 & -2.483516 & 8.122396 & 1.71192 \\
\hline 97 & 6 & 0 & -9.155745 & 0.025481 & -2.129139 \\
\hline 98 & 6 & 0 & -4.337031 & -4.870432 & -6.641579 \\
\hline 99 & 6 & 0 & 9.155745 & -0.025481 & -2.129139 \\
\hline 100 & 6 & 0 & 4.337031 & 4.870432 & -6.641579 \\
\hline 101 & 6 & 0 & -4.870432 & 4.337031 & 6.641579 \\
\hline 102 & 6 & 0 & 0.025481 & 9.155745 & 2.129139 \\
\hline 103 & 6 & 0 & 4.870432 & -4.337031 & 6.641579 \\
\hline 104 & 6 & 0 & -0.025481 & -9.155745 & 2.129139 \\
\hline 105 & 34 & 0 & -3.694534 & 0.167653 & 1.693713 \\
\hline 106 & 34 & 0 & -1.346093 & -1.803649 & 0.682831 \\
\hline 107 & 34 & 0 & -0.167653 & -3.694534 & -1.693713 \\
\hline 108 & 34 & 0 & 1.803649 & -1.346093 & -0.68283 \\
\hline 109 & 34 & 0 & 3.694534 & -0.167653 & 1.69371 \\
\hline 110 & 34 & 0 & 1.346093 & 1.803649 & 0.68283 \\
\hline 111 & 34 & 0 & 0.167653 & 3.694534 & -1.693713 \\
\hline 112 & 34 & 0 & -1.803649 & 1.346093 & -0.682831 \\
\hline 113 & 6 & 0 & -0.464798 & 10.113381 & 1.01695 \\
\hline 114 & 1 & 0 & -0.203110 & 11.149962 & 1.26290 \\
\hline 115 & 1 & 0 & -0.002828 & 9.864915 & 0.054667 \\
\hline 116 & 1 & 0 & -1.551091 & 10.065785 & 0.887537 \\
\hline 117 & 6 & 0 & -0.639860 & 9.546557 & 3.47031 \\
\hline 118 & 1 & 0 & -1.731565 & 9.479456 & 3.418843 \\
\hline 119 & 1 & 0 & -0.302964 & 8.889785 & 4.280483 \\
\hline 120 & 1 & 0 & -0.381017 & 10.578296 & 3.738634 \\
\hline 121 & 6 & 0 & 1.548024 & 9.335472 & 2.27924 \\
\hline 122 & 1 & 0 & 1.957053 & 8.703949 & 3.07608 \\
\hline 123 & 1 & 0 & 2.080019 & 9.101238 & 1.350183 \\
\hline 124 & 1 & 0 & 1.773299 & 10.376994 & $2.5352 ?$ \\
\hline
\end{tabular}




\begin{tabular}{|c|c|c|c|c|c|}
\hline 125 & 6 & 0 & -9.335472 & 1.548024 & -2.279246 \\
\hline 126 & 1 & 0 & -9.101238 & 2.080019 & -1.350183 \\
\hline 127 & 1 & 0 & -8.703949 & 1.957053 & -3.076083 \\
\hline 128 & 1 & 0 & -10.376994 & 1.773299 & -2.535231 \\
\hline 129 & 6 & 0 & -10.113381 & -0.464798 & -1.016951 \\
\hline 130 & 1 & 0 & -11.149962 & -0.203110 & -1.262901 \\
\hline 131 & 1 & 0 & -10.065785 & -1.551091 & -0.887537 \\
\hline 132 & 1 & 0 & -9.864915 & -0.002828 & -0.054667 \\
\hline 133 & 6 & 0 & -9.546557 & -0.639860 & -3.470311 \\
\hline 134 & 1 & 0 & -8.889785 & -0.302964 & -4.280483 \\
\hline 135 & 1 & 0 & -9.479456 & -1.731565 & -3.418843 \\
\hline 136 & 1 & 0 & -10.578296 & -0.381017 & -3.738634 \\
\hline 137 & 6 & 0 & -1.548024 & -9.335472 & 2.279246 \\
\hline 138 & 1 & 0 & -1.957053 & -8.703949 & 3.076083 \\
\hline 139 & 1 & 0 & -2.080019 & -9.101238 & 1.350183 \\
\hline 140 & 1 & 0 & -1.773299 & -10.376994 & 2.535231 \\
\hline 141 & 6 & 0 & 0.464798 & -10.113381 & 1.016951 \\
\hline 142 & 1 & 0 & 0.203110 & -11.149962 & 1.262901 \\
\hline 143 & 1 & 0 & 0.002828 & -9.864915 & 0.054667 \\
\hline 144 & 1 & 0 & 1.551091 & -10.065785 & 0.887537 \\
\hline 145 & 6 & 0 & 0.639860 & -9.546557 & 3.470311 \\
\hline 146 & 1 & 0 & 1.731565 & -9.479456 & 3.418843 \\
\hline 147 & 1 & 0 & 0.302964 & -8.889785 & 4.280483 \\
\hline 148 & 1 & 0 & 0.381017 & -10.578296 & 3.738634 \\
\hline 149 & 6 & 0 & 4.643712 & -5.846708 & 6.894083 \\
\hline 150 & 1 & 0 & 3.579408 & -6.103960 & 6.899591 \\
\hline 151 & 1 & 0 & 5.129108 & -6.450737 & 6.118958 \\
\hline 152 & 1 & 0 & 5.062692 & -6.138396 & 7.865069 \\
\hline 153 & 6 & 0 & 6.384586 & -4.061813 & 6.708393 \\
\hline 154 & 1 & 0 & 6.937172 & -4.634263 & 5.954605 \\
\hline 155 & 1 & 0 & 6.612831 & -2.999346 & 6.566608 \\
\hline 156 & 1 & 0 & 6.769620 & -4.353698 & 7.692017 \\
\hline 157 & 6 & 0 & 4.182988 & -3.521926 & 7.762980 \\
\hline 158 & 1 & 0 & 4.334052 & -2.446683 & 7.614460 \\
\hline 159 & 1 & 0 & 3.104060 & -3.705399 & 7.797757 \\
\hline 160 & 1 & 0 & 4.599182 & -3.792563 & 8.741231 \\
\hline 161 & 6 & 0 & -4.182988 & 3.521926 & 7.762980 \\
\hline 162 & 1 & 0 & -4.334052 & 2.446683 & 7.614460 \\
\hline 163 & 1 & 0 & -3.104060 & 3.705399 & 7.797757 \\
\hline 164 & 1 & 0 & -4.599182 & 3.792563 & 8.741231 \\
\hline 165 & 6 & 0 & -4.643712 & 5.846708 & 6.894083 \\
\hline 166 & 1 & 0 & -3.579408 & 6.103960 & 6.899591 \\
\hline 167 & 1 & 0 & -5.129108 & 6.450737 & 6.118958 \\
\hline 168 & 1 & 0 & -5.062692 & 6.138396 & 7.865069 \\
\hline 169 & 6 & 0 & -6.384586 & 4.061813 & 6.708393 \\
\hline 170 & 1 & 0 & -6.937172 & 4.634263 & 5.954605 \\
\hline 171 & 1 & 0 & -6.612831 & 2.999346 & 6.566608 \\
\hline 172 & 1 & 0 & -6.769620 & 4.353698 & 7.692017 \\
\hline 173 & 6 & 0 & -3.521926 & -4.182988 & -7.762980 \\
\hline 174 & 1 & 0 & -3.705399 & -3.104060 & -7.797757 \\
\hline 175 & 1 & 0 & -2.446683 & -4.334052 & -7.614460 \\
\hline 176 & 1 & 0 & -3.792563 & -4.599182 & -8.741231 \\
\hline 177 & 6 & 0 & -5.846708 & -4.643712 & -6.894083 \\
\hline 178 & 1 & 0 & -6.450737 & -5.129108 & -6.118958 \\
\hline 179 & 1 & 0 & -6.103960 & -3.579408 & -6.899591 \\
\hline 180 & 1 & 0 & -6.138396 & -5.062692 & -7.865069 \\
\hline 181 & 6 & 0 & -4.061813 & -6.384586 & -6.708393 \\
\hline 182 & 1 & 0 & -2.999346 & -6.612831 & -6.566608 \\
\hline 183 & 1 & 0 & -4.634263 & -6.937172 & -5.954605 \\
\hline 184 & 1 & 0 & -4.353698 & -6.769620 & -7.692017 \\
\hline 185 & 6 & 0 & 3.521926 & 4.182988 & -7.762980 \\
\hline 186 & 1 & 0 & 3.705399 & 3.104060 & -7.797757 \\
\hline 187 & 1 & 0 & 2.446683 & 4.334052 & -7.614460 \\
\hline 188 & 1 & 0 & 3.792563 & 4.599182 & -8.741231 \\
\hline $1 \varepsilon$ & 6 & 0 & 5.846708 & 4.643712 & -6.894( \\
\hline
\end{tabular}




\begin{tabular}{llrrrr}
190 & 1 & 0 & 6.450737 & 5.129108 & -6.118958 \\
191 & 1 & 0 & 6.103960 & 3.579408 & -6.899591 \\
192 & 1 & 0 & 6.138396 & 5.062692 & -7.865069 \\
193 & 6 & 0 & 4.061813 & 6.384586 & -6.708393 \\
194 & 1 & 0 & 2.999346 & 6.612831 & -6.566608 \\
195 & 1 & 0 & 4.634263 & 6.937172 & -5.954605 \\
196 & 1 & 0 & 4.353698 & 6.769620 & -7.692017 \\
197 & 6 & 0 & 9.546557 & 0.639860 & -3.470311 \\
198 & 1 & 0 & 8.889785 & 0.302964 & -4.280483 \\
199 & 1 & 0 & 9.479456 & 1.731565 & -3.418843 \\
200 & 1 & 0 & 10.578296 & 0.381017 & -3.738634 \\
201 & 6 & 0 & 9.335472 & -1.548024 & -2.279246 \\
202 & 1 & 0 & 9.101238 & -2.080019 & -1.350183 \\
203 & 1 & 0 & 8.703949 & -1.957053 & -3.076083 \\
204 & 1 & 0 & 10.376994 & -1.773299 & -2.535231 \\
205 & 6 & 0 & 10.113381 & 0.464798 & -1.016951 \\
206 & 1 & 0 & 10.065785 & 1.551091 & -0.887537 \\
207 & 1 & 0 & 9.864915 & 0.002828 & -0.054667 \\
208 & 1 & 0 & 11.149962 & 0.203110 & -1.262901 \\
\hline----------------------------------------------------------------------------
\end{tabular}

Table S5. Atomic coordinate of optimized structure of 4-ii

\begin{tabular}{|c|c|c|c|c|c|}
\hline \multirow{2}{*}{$\begin{array}{l}\text { Center } \\
\text { Number }\end{array}$} & \multirow{2}{*}{$\begin{array}{l}\text { Atomic } \\
\text { Number }\end{array}$} & \multirow{2}{*}{$\begin{array}{l}\text { Atomic } \\
\text { Type }\end{array}$} & \multicolumn{3}{|c|}{ Coordinates (Angstroms) } \\
\hline & & & $\mathrm{X}$ & $\mathrm{Y}$ & $\mathrm{Z}$ \\
\hline 1 & 34 & 0 & 2.964729 & 3.075966 & -1.263432 \\
\hline 2 & 34 & 0 & 0.066169 & 2.658199 & -2.668525 \\
\hline 3 & 34 & 0 & 2.064186 & -0.108304 & -1.326496 \\
\hline 4 & 34 & 0 & -0.080949 & -3.262685 & -1.925339 \\
\hline 5 & 34 & 0 & -3.158834 & 2.679307 & -2.367654 \\
\hline 6 & 34 & 0 & -2.067390 & -0.234303 & -1.287370 \\
\hline 7 & 34 & 0 & 3.137039 & -3.201502 & -1.690791 \\
\hline 8 & 34 & 0 & -2.962288 & -3.310998 & -0.461971 \\
\hline 9 & 6 & 0 & 0.302460 & 3.064588 & 0.002235 \\
\hline 10 & 6 & 0 & -1.122017 & 3.046094 & -0.264657 \\
\hline 11 & 6 & 0 & 4.704619 & -0.731367 & -1.670818 \\
\hline 12 & 6 & 0 & -0.869588 & -2.797921 & 2.118424 \\
\hline 13 & 6 & 0 & 5.852449 & 1.601599 & -1.656857 \\
\hline 14 & 6 & 0 & 6.117226 & 2.560429 & -0.668873 \\
\hline 15 & 1 & 0 & 5.442012 & 2.651513 & 0.177676 \\
\hline 16 & 6 & 0 & 4.659956 & 0.719546 & -1.562703 \\
\hline 17 & 6 & 0 & -2.172916 & 3.138604 & 0.780312 \\
\hline 18 & 6 & 0 & -3.389557 & -1.564446 & -1.099448 \\
\hline 19 & 6 & 0 & 7.223579 & 3.407919 & -0.761385 \\
\hline 20 & 1 & 0 & 7.380588 & 4.131981 & 0.030533 \\
\hline 21 & 6 & 0 & 3.396071 & 1.223171 & -1.390050 \\
\hline 22 & 6 & 0 & -0.291069 & -2.955257 & 0.759958 \\
\hline 23 & 6 & 0 & 3.476198 & -1.331181 & -1.572082 \\
\hline 24 & 6 & 0 & -4.714904 & 0.297349 & -1.723751 \\
\hline 25 & 6 & 0 & 8.117979 & 3.330699 & -1.836033 \\
\hline 26 & 6 & 0 & 5.951160 & -1.520932 & -1.834024 \\
\hline 27 & 6 & 0 & 6.075787 & -2.461443 & -2.869891 \\
\hline 28 & 1 & 0 & 5.263348 & -2.578784 & -3.583169 \\
\hline 29 & 6 & 0 & -4.658583 & -1.108793 & -1.352666 \\
\hline 30 & 6 & 0 & 1.132580 & -2.999622 & 0.482947 \\
\hline 31 & 6 & 0 & 2.097448 & 3.676110 & 3.898879 \\
\hline 32 & 6 & 0 & 7.228795 & -3.230248 & -3.007600 \\
\hline 33 & 1 & 0 & 7.279025 & -3.943638 & -3.825724 \\
\hline 34 & 6 & 0 & -5.965798 & 1.008284 & -2.102014 \\
\hline
\end{tabular}




\begin{tabular}{|c|c|c|c|c|c|}
\hline 35 & 6 & 0 & -6.805711 & 0.520091 & -3.115467 \\
\hline 36 & 1 & 0 & -6.540609 & -0.396628 & -3.633838 \\
\hline 37 & 6 & 0 & -1.437443 & 2.867661 & -1.596185 \\
\hline 38 & 6 & 0 & 0.894244 & 3.266058 & 1.348799 \\
\hline 39 & 6 & 0 & 8.315416 & -3.094735 & -2.128695 \\
\hline 40 & 6 & 0 & 2.453263 & 2.587935 & 3.082682 \\
\hline 41 & 1 & 0 & 3.204636 & 1.879611 & 3.420551 \\
\hline 42 & 6 & 0 & 1.872624 & 2.382825 & 1.835611 \\
\hline 43 & 1 & 0 & 2.157111 & 1.522037 & 1.238673 \\
\hline 44 & 6 & 0 & 1.117146 & 4.546605 & 3.404511 \\
\hline 45 & 1 & 0 & 0.801172 & 5.403243 & 3.989373 \\
\hline 46 & 6 & 0 & -8.129476 & -3.682763 & -0.931572 \\
\hline 47 & 6 & 0 & -5.920819 & -3.220916 & -1.869055 \\
\hline 48 & 1 & 0 & -5.088588 & -3.536456 & -2.492272 \\
\hline 49 & 6 & 0 & 0.524142 & 4.346169 & 2.157281 \\
\hline 50 & 1 & 0 & -0.229159 & 5.045647 & 1.807359 \\
\hline 51 & 6 & 0 & 7.847708 & 2.362441 & -2.819882 \\
\hline 52 & 1 & 0 & 8.508976 & 2.264436 & -3.676620 \\
\hline 53 & 6 & 0 & -9.337831 & -4.627549 & -0.804119 \\
\hline 54 & 6 & 0 & -5.853845 & -1.980836 & -1.211188 \\
\hline 55 & 6 & 0 & 6.746882 & 1.517431 & -2.736677 \\
\hline 56 & 1 & 0 & 6.572438 & 0.785261 & -3.519213 \\
\hline 57 & 6 & 0 & 2.188291 & -2.801227 & 1.508495 \\
\hline 58 & 6 & 0 & -7.030584 & -4.048957 & -1.726499 \\
\hline 59 & 1 & 0 & -7.037211 & -4.998041 & -2.255700 \\
\hline 60 & 6 & 0 & -6.345526 & 2.192800 & -1.455217 \\
\hline 61 & 1 & 0 & -5.713843 & 2.596300 & -0.669088 \\
\hline 62 & 6 & 0 & -2.208496 & 2.236091 & 1.848287 \\
\hline 63 & 1 & 0 & -1.441118 & 1.472848 & 1.931794 \\
\hline 64 & 6 & 0 & -7.970498 & 1.195065 & -3.465320 \\
\hline 65 & 1 & 0 & -8.587538 & 0.781787 & -4.258648 \\
\hline 66 & 6 & 0 & -8.359485 & 2.384175 & -2.824185 \\
\hline 67 & 6 & 0 & -4.238706 & 3.259081 & 2.740224 \\
\hline 68 & 6 & 0 & -0.485907 & -3.627668 & 3.177724 \\
\hline 69 & 1 & 0 & 0.266586 & -4.393406 & 3.014646 \\
\hline 70 & 6 & 0 & 2.772105 & 3.866385 & 5.269093 \\
\hline 71 & 6 & 0 & -3.492168 & 0.914587 & -1.735693 \\
\hline 72 & 6 & 0 & 9.573692 & -3.960825 & -2.318680 \\
\hline 73 & 6 & 0 & -8.057048 & -2.441799 & -0.283120 \\
\hline 74 & 1 & 0 & -8.874683 & -2.106289 & 0.345345 \\
\hline 75 & 6 & 0 & 7.033558 & -1.377026 & -0.956067 \\
\hline 76 & 1 & 0 & 6.970483 & -0.658696 & -0.144272 \\
\hline 77 & 6 & 0 & -3.189707 & 4.102818 & 0.699882 \\
\hline 78 & 1 & 0 & -3.192724 & 4.808730 & -0.124616 \\
\hline 79 & 6 & 0 & -7.517094 & 2.863172 & -1.812181 \\
\hline 80 & 1 & 0 & -7.767662 & 3.773461 & -1.278394 \\
\hline 81 & 6 & 0 & 3.235117 & -3.723249 & 1.662947 \\
\hline 82 & 1 & 0 & 3.262453 & -4.611661 & 1.040283 \\
\hline 83 & 6 & 0 & -5.366581 & 3.362498 & 3.782103 \\
\hline 84 & 6 & 0 & 4.236870 & -3.512141 & 2.604182 \\
\hline 85 & 1 & 0 & 5.024644 & -4.254997 & 2.693681 \\
\hline 86 & 6 & 0 & 9.348171 & 4.245309 & -1.972469 \\
\hline 87 & 6 & 0 & -4.195874 & 4.157539 & 1.659341 \\
\hline 88 & 1 & 0 & -4.957710 & 4.926705 & 1.564946 \\
\hline 89 & 6 & 0 & -2.040384 & -2.523238 & 4.701179 \\
\hline 90 & 6 & 0 & 1.433880 & -3.175671 & -0.852411 \\
\hline 91 & 6 & 0 & -3.219123 & 2.300697 & 2.808972 \\
\hline 92 & 1 & 0 & -3.196397 & 1.582538 & 3.620873 \\
\hline 93 & 6 & 0 & -1.845685 & -1.818551 & 2.367732 \\
\hline
\end{tabular}




\begin{tabular}{|c|c|c|c|c|c|}
\hline 94 & 1 & 0 & -2.144030 & -1.148521 & 1.567353 \\
\hline 95 & 6 & 0 & -6.947117 & -1.607885 & -0.418572 \\
\hline 96 & 1 & 0 & -6.929531 & -0.654517 & 0.100876 \\
\hline 97 & 6 & 0 & -1.081390 & -3.093319 & -0.364838 \\
\hline 98 & 6 & 0 & -1.062952 & -3.493044 & 4.440950 \\
\hline 99 & 1 & 0 & -0.736902 & -4.165559 & 5.226539 \\
\hline 100 & 6 & 0 & 4.249840 & -2.375264 & 3.429961 \\
\hline 101 & 6 & 0 & -10.437832 & -4.052629 & 0.108293 \\
\hline 102 & 1 & 0 & -10.074870 & -3.884353 & 1.128617 \\
\hline 103 & 1 & 0 & -11.274090 & -4.758439 & 0.167731 \\
\hline 104 & 1 & 0 & -10.832298 & -3.104839 & -0.275404 \\
\hline 105 & 6 & 0 & -9.951939 & -4.870376 & -2.203517 \\
\hline 106 & 1 & 0 & -10.297662 & -3.930093 & -2.648038 \\
\hline 107 & 1 & 0 & -10.811030 & -5.548738 & -2.131009 \\
\hline 108 & 1 & 0 & -9.229986 & -5.320054 & -2.893173 \\
\hline 109 & 6 & 0 & 9.476323 & 5.233987 & -0.798172 \\
\hline 110 & 1 & 0 & 8.608541 & 5.899665 & -0.728808 \\
\hline 111 & 1 & 0 & 10.363448 & 5.861746 & -0.939349 \\
\hline 112 & 1 & 0 & 9.587840 & 4.715869 & 0.161189 \\
\hline 113 & 6 & 0 & 1.082390 & 2.898621 & -1.126600 \\
\hline 114 & 6 & 0 & 8.186134 & -2.148270 & -1.101505 \\
\hline 115 & 1 & 0 & 8.992351 & -2.001191 & -0.391288 \\
\hline 116 & 6 & 0 & -2.410551 & -1.688935 & 3.632059 \\
\hline 117 & 1 & 0 & -3.163659 & -0.919946 & 3.779923 \\
\hline 118 & 6 & 0 & -8.874821 & -5.978155 & -0.207640 \\
\hline 119 & 1 & 0 & -8.118758 & -6.462676 & -0.834196 \\
\hline 120 & 1 & 0 & -9.724133 & -6.666570 & -0.116751 \\
\hline 121 & 1 & 0 & -8.441895 & -5.838170 & 0.789394 \\
\hline 122 & 6 & 0 & -9.658062 & 3.096147 & -3.243241 \\
\hline 123 & 6 & 0 & 2.194670 & -1.661918 & 2.321149 \\
\hline 124 & 1 & 0 & 1.403959 & -0.925329 & 2.219343 \\
\hline 125 & 6 & 0 & -2.699359 & -2.347436 & 6.080897 \\
\hline 126 & 6 & 0 & 3.203146 & -1.458595 & 3.264129 \\
\hline 127 & 1 & 0 & 3.155742 & -0.564152 & 3.875129 \\
\hline 128 & 6 & 0 & 5.380199 & -2.184584 & 4.456645 \\
\hline 129 & 6 & 0 & -9.912858 & 4.378515 & -2.428619 \\
\hline 130 & 1 & 0 & -10.015114 & 4.168163 & -1.357852 \\
\hline 131 & 1 & 0 & -10.844393 & 4.849219 & -2.762874 \\
\hline 132 & 1 & 0 & -9.107831 & 5.110782 & -2.558332 \\
\hline 133 & 6 & 0 & -5.303959 & 4.746993 & 4.470551 \\
\hline 134 & 1 & 0 & -4.347327 & 4.884380 & 4.987452 \\
\hline 135 & 1 & 0 & -6.107761 & 4.842350 & 5.210954 \\
\hline 136 & 1 & 0 & -5.414918 & 5.564728 & 3.750705 \\
\hline 137 & 6 & 0 & -5.259301 & 2.279519 & 4.872194 \\
\hline 138 & 1 & 0 & -5.324294 & 1.269838 & 4.450419 \\
\hline 139 & 1 & 0 & -6.082546 & 2.391845 & 5.586684 \\
\hline 140 & 1 & 0 & -4.321982 & 2.357251 & 5.434862 \\
\hline 141 & 6 & 0 & 9.237883 & 5.063827 & -3.281060 \\
\hline 142 & 1 & 0 & 9.184912 & 4.417192 & -4.163140 \\
\hline 143 & 1 & 0 & 10.111770 & 5.716657 & -3.397789 \\
\hline 144 & 1 & 0 & 8.341008 & 5.693581 & -3.274589 \\
\hline 145 & 6 & 0 & 10.631056 & 3.381282 & -2.016526 \\
\hline 146 & 1 & 0 & 10.740902 & 2.795917 & -1.096412 \\
\hline 147 & 1 & 0 & 11.517384 & 4.019044 & -2.122046 \\
\hline 148 & 1 & 0 & 10.622439 & 2.681027 & -2.858283 \\
\hline 149 & 6 & 0 & -9.572581 & 3.485306 & -4.738455 \\
\hline 150 & 1 & 0 & -8.733928 & 4.167554 & -4.918215 \\
\hline 151 & 1 & 0 & -10.494652 & 3.988053 & -5.055563 \\
\hline 152 & 1 & 0 & -9.433128 & 2.609287 & -5.380400 \\
\hline
\end{tabular}




\begin{tabular}{|c|c|c|c|c|c|}
\hline 153 & 6 & 0 & -10.859464 & 2.144441 & -3.030671 \\
\hline 154 & 1 & 0 & -10.760203 & 1.225519 & -3.617762 \\
\hline 155 & 1 & 0 & -11.793278 & 2.634600 & -3.332800 \\
\hline 156 & 1 & 0 & -10.950832 & 1.858630 & -1.976500 \\
\hline 157 & 6 & 0 & 5.228320 & -0.880533 & 5.262154 \\
\hline 158 & 1 & 0 & 5.255975 & 0.003349 & 4.614278 \\
\hline 159 & 1 & 0 & 6.053305 & -0.789837 & 5.977783 \\
\hline 160 & 1 & 0 & 4.292747 & -0.859074 & 5.832713 \\
\hline 161 & 6 & 0 & 2.483302 & 2.635242 & 6.160819 \\
\hline 162 & 1 & 0 & 1.406040 & 2.508962 & 6.317847 \\
\hline 163 & 1 & 0 & 2.959198 & 2.754420 & 7.142022 \\
\hline 164 & 1 & 0 & 2.866673 & 1.711171 & 5.715491 \\
\hline 165 & 6 & 0 & 6.740732 & -2.139421 & 3.720808 \\
\hline 166 & 1 & 0 & 6.932404 & -3.060578 & 3.161066 \\
\hline 167 & 1 & 0 & 7.559252 & -2.007653 & 4.439322 \\
\hline 168 & 1 & 0 & 6.773344 & -1.305855 & 3.009925 \\
\hline 169 & 6 & 0 & 4.300490 & 4.010663 & 5.076010 \\
\hline 170 & 1 & 0 & 4.736180 & 3.128200 & 4.595858 \\
\hline 171 & 1 & 0 & 4.796778 & 4.141674 & 6.045491 \\
\hline 172 & 1 & 0 & 4.536209 & 4.880714 & 4.452641 \\
\hline 173 & 6 & 0 & 2.262256 & 5.120294 & 6.004663 \\
\hline 174 & 1 & 0 & 2.459272 & 6.035613 & 5.435061 \\
\hline 175 & 1 & 0 & 2.773251 & 5.215210 & 6.969455 \\
\hline 176 & 1 & 0 & 1.186181 & 5.066793 & 6.205226 \\
\hline 177 & 6 & 0 & 5.376396 & -3.368263 & 5.453286 \\
\hline 178 & 1 & 0 & 4.426061 & -3.419342 & 5.996911 \\
\hline 179 & 1 & 0 & 6.182997 & -3.252182 & 6.187723 \\
\hline 180 & 1 & 0 & 5.522877 & -4.327682 & 4.946348 \\
\hline 181 & 6 & 0 & -2.172722 & -3.359959 & 7.115652 \\
\hline 182 & 1 & 0 & -2.370558 & -4.394044 & 6.811424 \\
\hline 183 & 1 & 0 & -2.671826 & -3.197582 & 8.077693 \\
\hline 184 & 1 & 0 & -1.094686 & -3.251906 & 7.280769 \\
\hline 185 & 6 & 0 & -4.228545 & -2.544339 & 5.950583 \\
\hline 186 & 1 & 0 & -4.675439 & -1.822893 & 5.258622 \\
\hline 187 & 1 & 0 & -4.714028 & -2.416266 & 6.925976 \\
\hline 188 & 1 & 0 & -4.464132 & -3.549156 & 5.582106 \\
\hline 189 & 6 & 0 & 9.189877 & -5.456407 & -2.218025 \\
\hline 190 & 1 & 0 & 8.761514 & -5.685603 & -1.235603 \\
\hline 191 & 1 & 0 & 10.075479 & -6.088253 & -2.359217 \\
\hline 192 & 1 & 0 & 8.453627 & -5.740414 & -2.977135 \\
\hline 193 & 6 & 0 & 10.651205 & -3.670136 & -1.256816 \\
\hline 194 & 1 & 0 & 10.991220 & -2.628961 & -1.294529 \\
\hline 195 & 1 & 0 & 11.524132 & -4.308980 & -1.432514 \\
\hline 196 & 1 & 0 & 10.290475 & -3.875829 & -0.242562 \\
\hline 197 & 6 & 0 & 10.184725 & -3.682664 & -3.712816 \\
\hline 198 & 1 & 0 & 9.480525 & -3.911342 & -4.519583 \\
\hline 199 & 1 & 0 & 11.079872 & -4.297764 & -3.867121 \\
\hline 200 & 1 & 0 & 10.474350 & -2.630151 & -3.810499 \\
\hline 201 & 6 & 0 & -2.410422 & -0.923259 & 6.612393 \\
\hline 202 & 1 & 0 & -1.332603 & -0.755340 & 6.718473 \\
\hline 203 & 1 & 0 & -2.876039 & -0.780841 & 7.595441 \\
\hline 204 & 1 & 0 & -2.804500 & -0.151350 & 5.942936 \\
\hline 205 & 6 & 0 & -6.735810 & 3.202412 & 3.079343 \\
\hline 206 & 1 & 0 & -6.900272 & 3.978454 & 2.324563 \\
\hline 207 & 1 & 0 & -7.550325 & 3.272021 & 3.810791 \\
\hline 208 & 1 & 0 & -6.809050 & 2.229984 & 2.579282 \\
\hline
\end{tabular}


S6. Molecular Orbitals

(a)
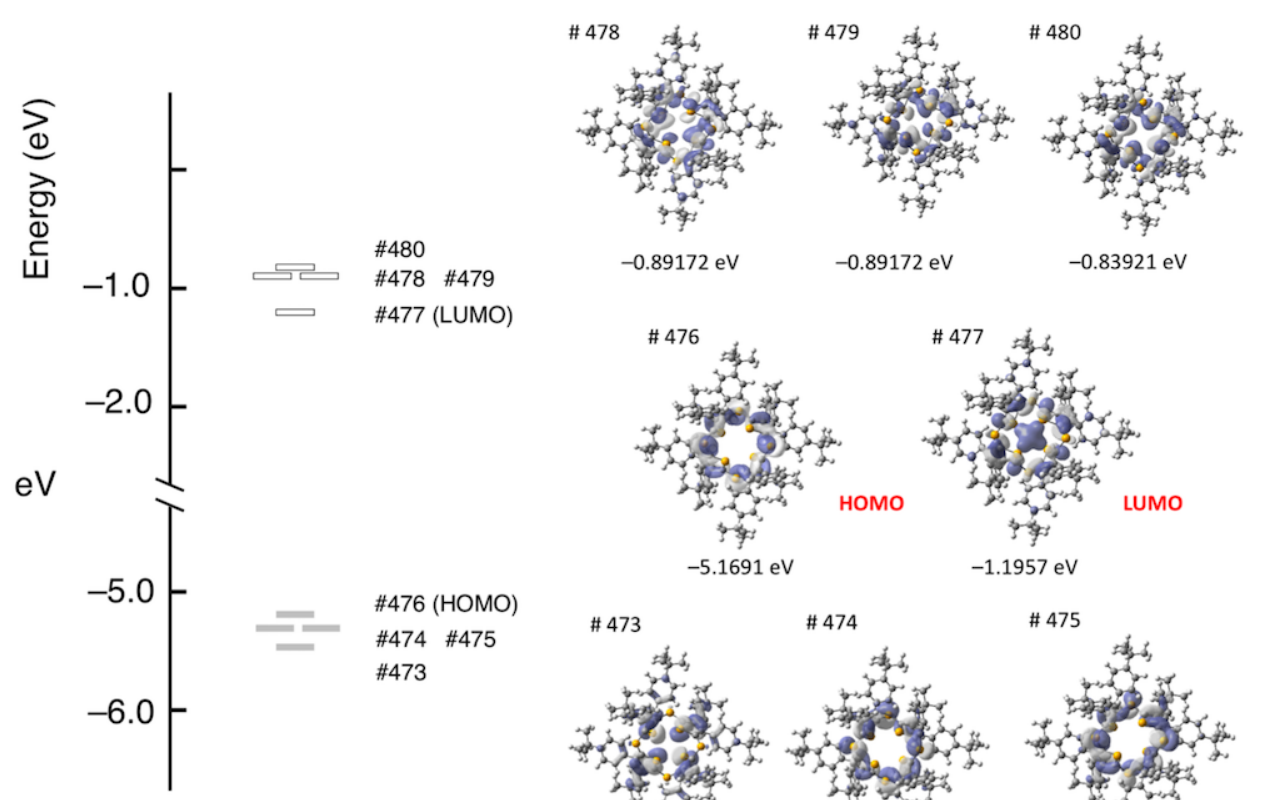

$$
-5.1691 \mathrm{eV} \quad-1.1957 \mathrm{eV}
$$

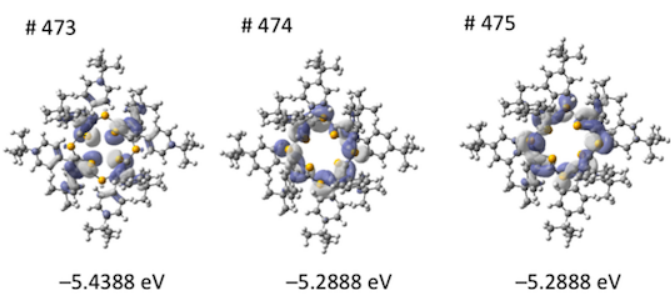

(b)
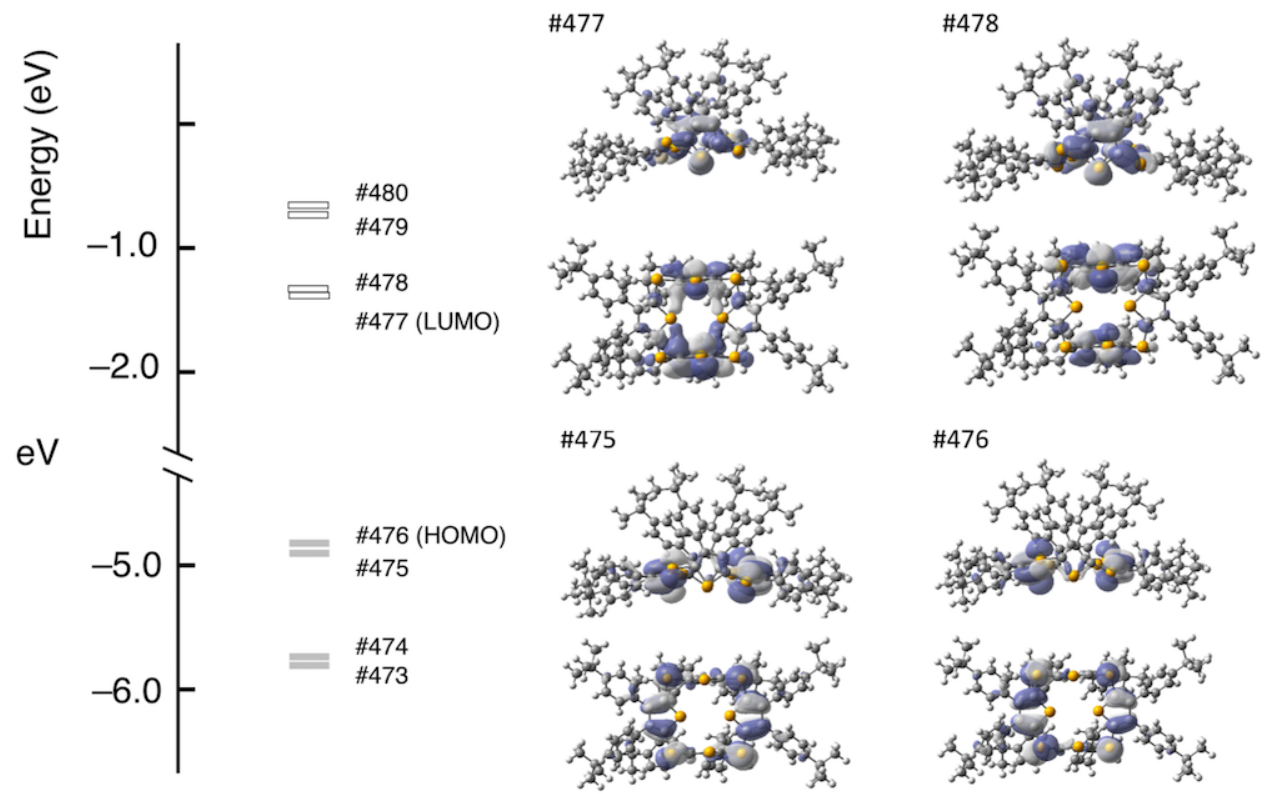

Figure S6. Molecular orbitals and their energy of (a) 4-i ( $\left.S_{4}\right)$ and (b) 4-ii $\left(C_{1}\right)$ 


\section{S7. TD-DFT Calculations}

The optimized $S_{4}$ (B3LYP/6-31G(d)) structure of 4-i was applied for TD-DFT calculations. Selected excitation energies and their transition nature are listed as follow.

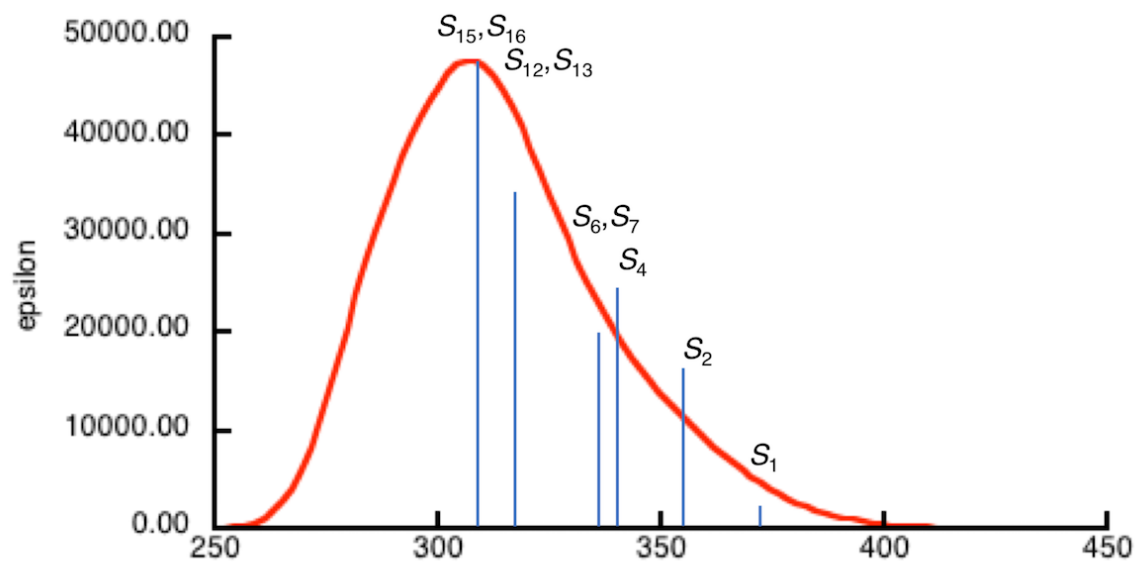

Figure S7. Simulated electronic spectra of 4-ifrom TD-DFT calculation (isovalue : 0.15).

Table S6. Selected electronic transition for 4

\begin{tabular}{cccc}
\hline State & $\begin{array}{c}\text { Excitation energies } \\
\text { (Oscillator strengths })\end{array}$ & \multicolumn{2}{c}{ Nature } \\
\hline$S_{1}$ & $3.3272 \mathrm{eV}, 372.64 \mathrm{~nm}(f=0.0000)$ & 476 -> 477 & 0.67757 \\
\hline$S_{2}$ & $3.4898 \mathrm{eV}, 355.28 \mathrm{~nm}(f=0.0432)$ & 475 - 477 & 0.43752 \\
\hline$S_{4}$ & $3.6885 \mathrm{eV}, 336.14 \mathrm{~nm}(f=0.0627)$ & $473->477$ & 0.67843 \\
\hline$S_{6}$ & $3.7283 \mathrm{eV}, 332.55 \mathrm{~nm}(f=0.0472)$ & $476->479$ & 0.60315 \\
\hline$S_{7}$ & $3.7283 \mathrm{eV}, 332.55 \mathrm{~nm}(f=0.0472)$ & $476->478$ & 0.60315 \\
\hline$S_{12}$ & $3.9116 \mathrm{eV}, 316.96 \mathrm{~nm}(f=0.0957)$ & $476->478$ & 0.57302 \\
\hline$S_{13}$ & $3.9116 \mathrm{eV}, 316.96 \mathrm{~nm}(f=0.0957)$ & $474->480$ & 0.57302 \\
\hline$S_{15}$ & $4.0092 \mathrm{eV}, 309.25 \mathrm{~nm}(f=0.1325)$ & $473->479$ & 0.58760 \\
\hline$S_{16}$ & $4.0092 \mathrm{eV}, 309.25 \mathrm{~nm}(f=0.1325)$ & $473->478$ & 0.58760 \\
\hline
\end{tabular}


S8. Cyclic Voltammetry

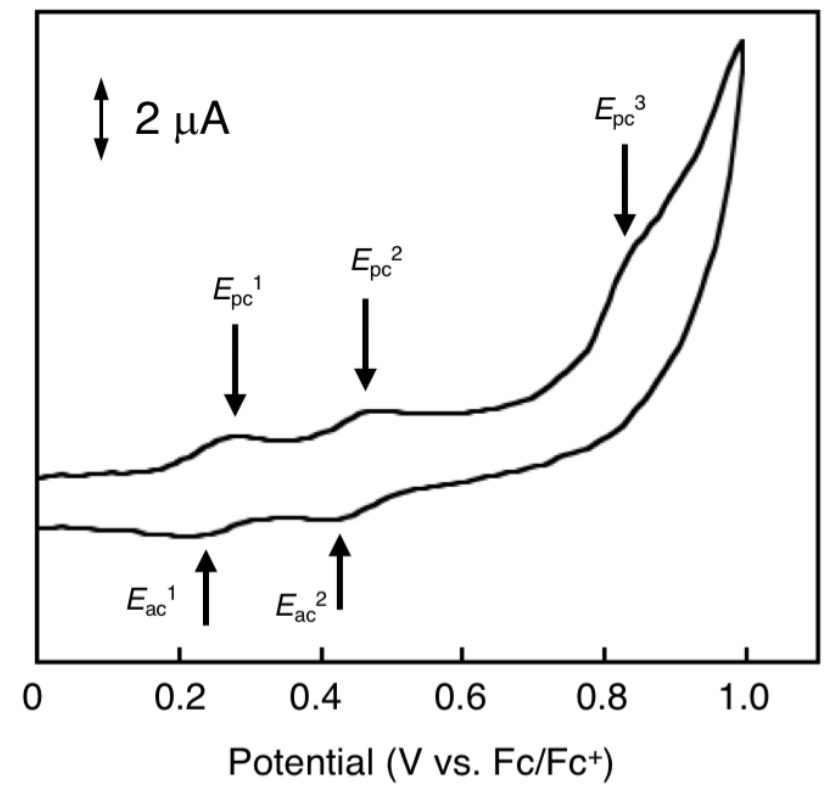

Figure S8. Cyclic voltammogram of 4 in $\mathrm{CH}_{2} \mathrm{Cl}_{2}$ containing $0.1 \mathrm{M}$ of $\mathrm{Bu}_{4} \mathrm{NPF}$.

\section{S9. Gelation Properties of 4 in Various Solvents}

Table S7. Gelation properties of 4 in Selected Solvents ${ }^{a}$

\begin{tabular}{cccc}
\hline Solvent & $\mathbf{4}$ & Solvent & $\mathbf{4}$ \\
\hline toluene & $\mathrm{G}\left(15 \mathrm{wt}^{b}\right)$ & THF & $\mathrm{S}$ \\
fluorobenzene & $\mathrm{PG}$ & 1,4-dioxane & $\mathrm{S}$ \\
chlorobenzene & $\mathrm{PG}$ & Hexane & $\mathrm{I}$ \\
benzene & $\mathrm{S}$ & Methylcyclohexane & $\mathrm{I}$ \\
$\mathrm{CHCl}_{3}$ & $\mathrm{~S}$ & Acetone & $\mathrm{I}$ \\
$\mathrm{CH}_{2} \mathrm{Cl}_{2}$ & $\mathrm{~S}$ & $\mathrm{MeOH}$ & $\mathrm{I}$
\end{tabular}

${ }^{a} \mathrm{I}=$ insoluble, $\mathrm{S}=$ solution even at $40 \mathrm{mM}, \mathrm{G}=\mathrm{Gel}, \mathrm{PG}=$ partially jelly precipitate. ${ }^{b} \mathrm{CGC}$ value. ${ }^{\text {In }}$ $3 \mathrm{mM}$ at rt. 


\section{S10. SEM Measurements}

Scanning electron microscopy (SEM) of xerogel from hot toluene solution were carried out on a KEYENCE VE-8800 microscope. Silicon wafers were used for a substrate. Images were recorded with appropriated working distance and acceleration voltage in each measurement.
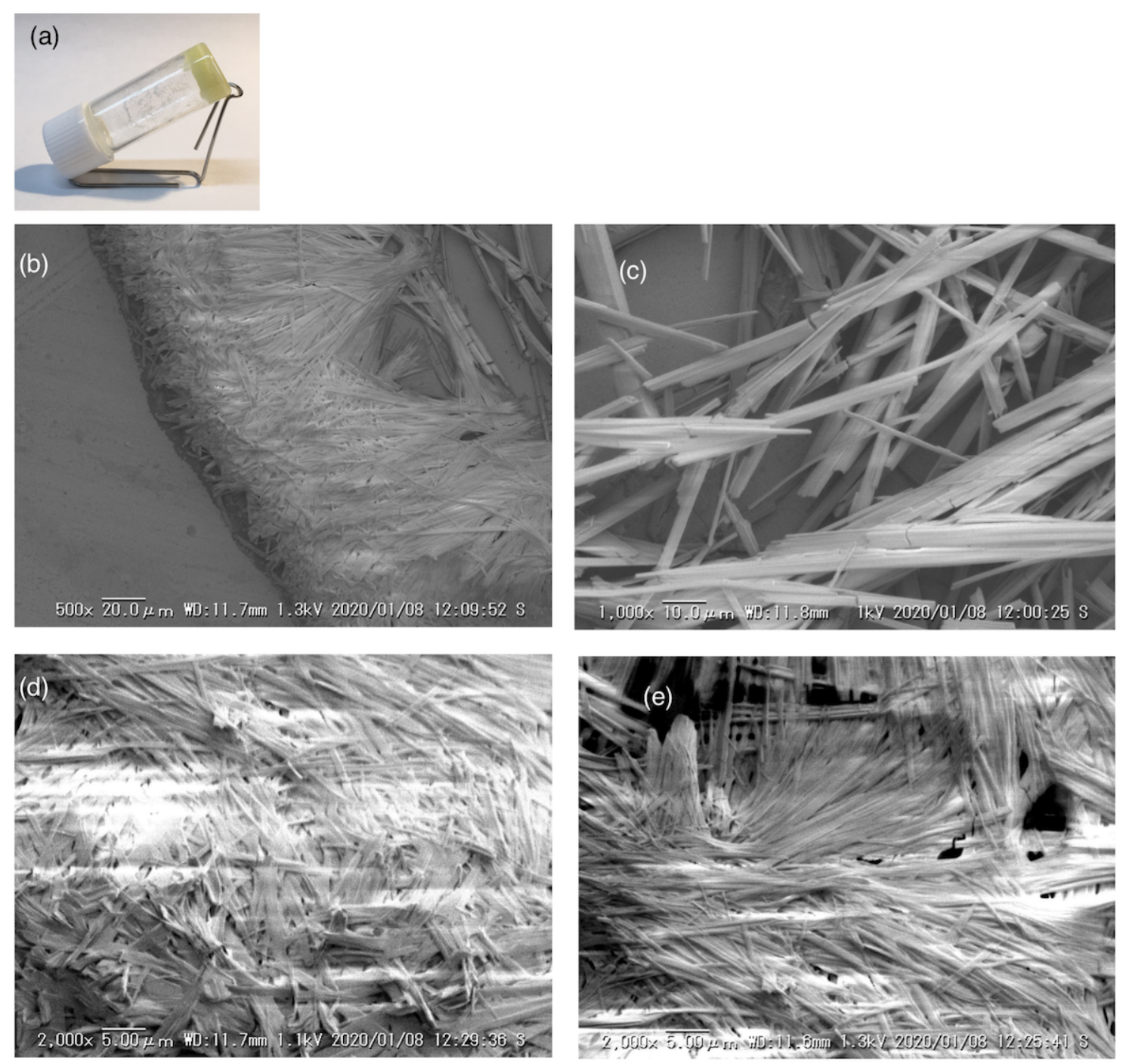

Figure S9. (a) Photograph of gel of 4 from toluene solution and (b)-(e) SEM images of its air-dried xerogel. 


\section{S11. XRD Analysis of Gel and Simulated Powder Pattern of Single Crystal}

$\mathrm{X}$-ray diffraction (XRD) analysis of xerogel obtained from hot toluene solution was carried out with Rigaku SmartLab X-ray Diffractometer with a capillary attachment. The data were collected in a capillary tube $(\mathrm{OD}=0.5 \mathrm{~mm}$, thickness $=0.01 \mathrm{~mm})$, purchased from Higenberg Co. Ltd, using standard $\theta / 2 \theta$ techniques, with monochromated $\mathrm{CuK} \alpha$ radiation $(\lambda=1.5418 \AA)$. A simulated powder patterns was generated by Mercury Software Ver. 4.00.

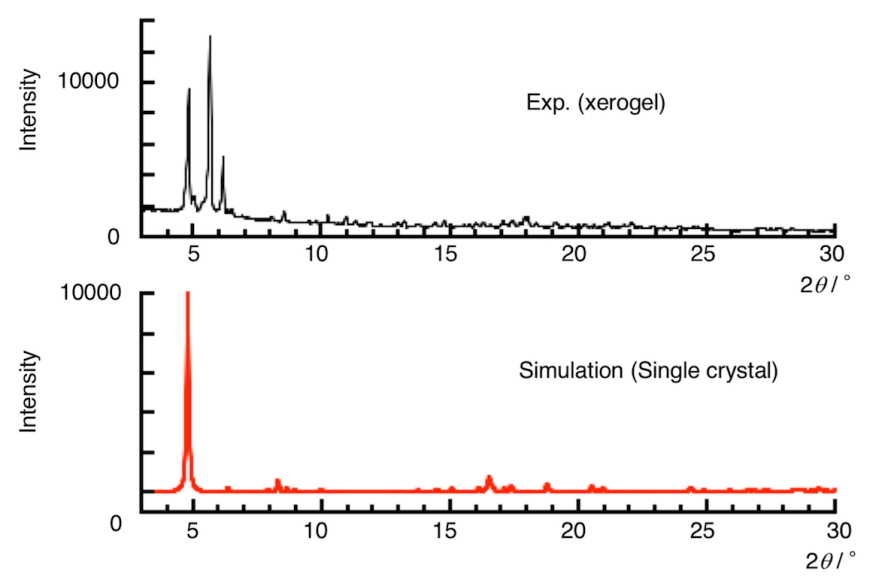

Figure S10. (a) XRD analysis of 4 in xerogel and (b) Simulated powder pattern of 4 in single crystal

Table S8. Selected diffraction peaks in gel (experimental) and single crystal (simulation)

\begin{tabular}{|c|c|c|c|c|c|}
\hline \multicolumn{3}{|c|}{ Gel (experimental) } & \multicolumn{3}{|c|}{ Single crystal (simulation) } \\
\hline $2 \theta\left(^{\circ}\right)$ & $d(\AA)$ & Intensity & $2 \theta\left(^{\circ}\right)$ & $d(\AA)$ & Plane \\
\hline 4.80 & 18.41 & 5504.8 & 4.81 & 18.37 & $(011)$ \\
\hline 5.00 & 17.67 & 661.5 & 4.83 & 18.31 & $(020)$ \\
\hline 5.63 & 15.67 & 7904.6 & 6.37 & 13.87 & $(021)$ \\
\hline 6.14 & 14.38 & 2622.8 & 7.43 & 11.9 & $(100)$ \\
\hline 6.46 & 13.66 & 250.2 & 7.81 & 11.3 & (110) \\
\hline 8.51 & 10.38 & 409.5 & 7.94 & 11.1 & $(11-1)$ \\
\hline 10.22 & 8.65 & 483.9 & 8.33 & 10.62 & $(002)$ \\
\hline 10.94 & 8.08 & 415.1 & 8.67 & 10.20 & $(031)$ \\
\hline 14.75 & 56.00 & 251.6 & 8.86 & 9.98 & $(120)$ \\
\hline 16.26 & 5.45 & 216.1 & 8.98 & 9.85 & $(12-1)$ \\
\hline 17.03 & 5.20 & 232.6 & 10.0 & 8.85 & $(11-2)$ \\
\hline 17.38 & 5.10 & 290 & 11.9 & 7.47 & $(131)$ \\
\hline 17.68 & 5.01 & 200 & 12.74 & 6.95 & (013) \\
\hline 17.87 & 4.96 & 473 & 13.10 & 6.76 & $(11-3)$ \\
\hline 18.00 & 4.93 & 540.6 & 13.76 & 6.44 & $(12-3)$ \\
\hline
\end{tabular}


S12. Figure S11. ${ }^{1} \mathrm{H}$ and ${ }^{13} \mathrm{C}$ NMR Charts

(a) ${ }^{1} \mathrm{H}$ NMR chart of $4\left(400 \mathrm{MHz}, \mathrm{CDCl}_{3}\right)$

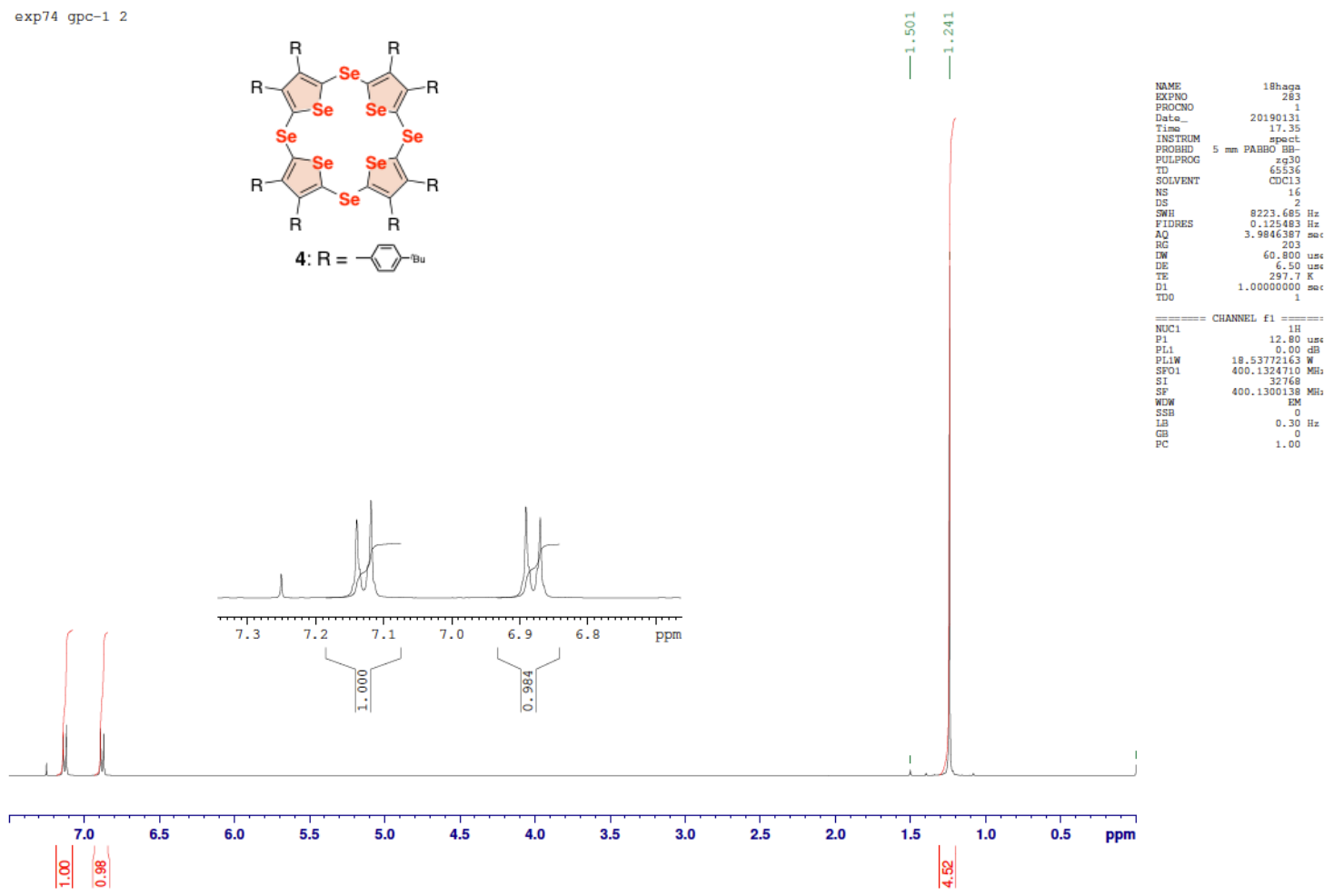

(b) ${ }^{13} \mathrm{C}$ NMR chart of $4\left(100 \mathrm{MHz}, \mathrm{CDCl}_{3}\right)$
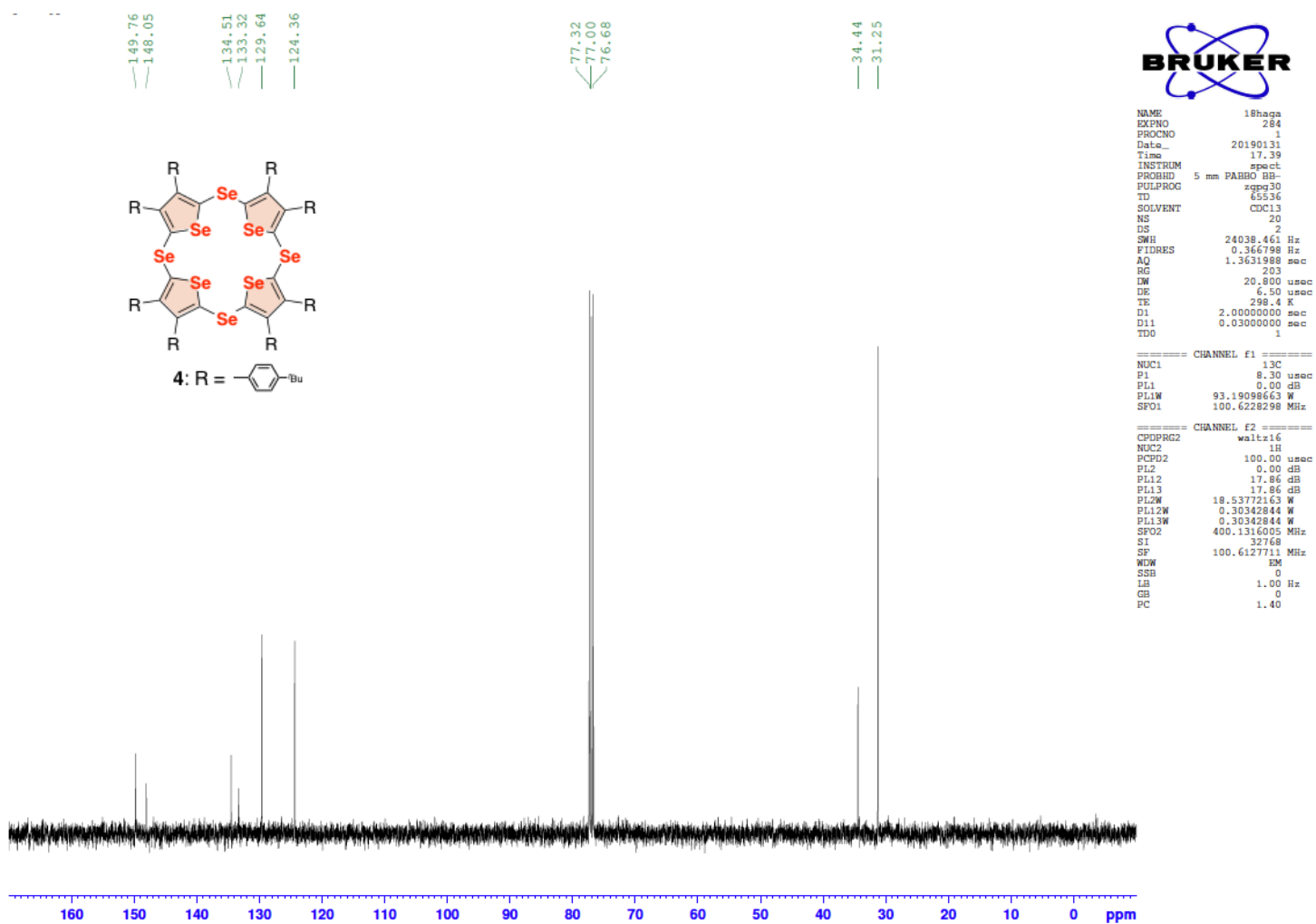
(c) ${ }^{77} \mathrm{Se}$ NMR chart of $4\left(76 \mathrm{MHz}, \mathrm{CDCl}_{3}\right)$
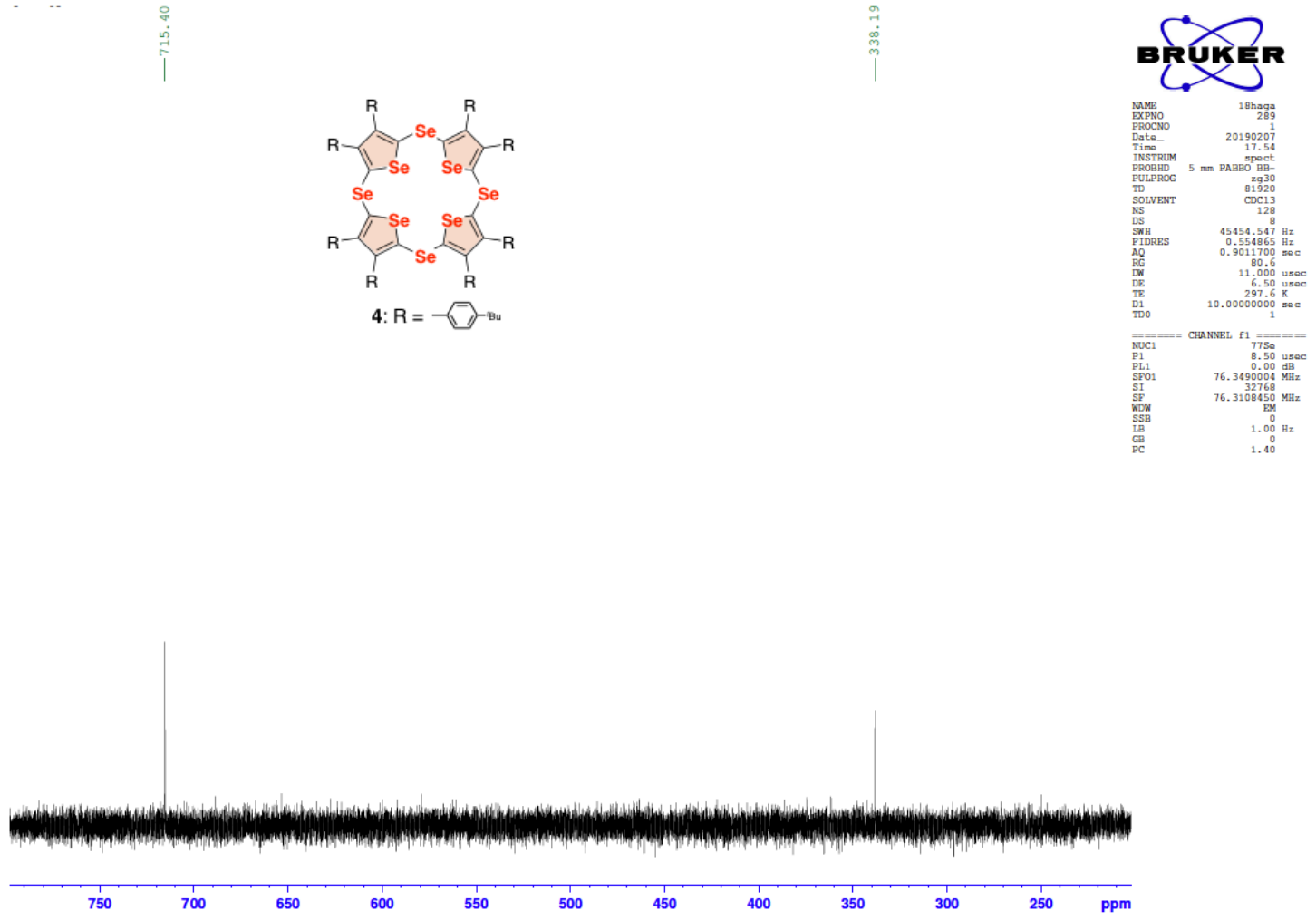

(d) ${ }^{1} \mathrm{H}$ NMR chart of $7\left(400 \mathrm{MHz}, \mathrm{CDCl}_{3}\right)$

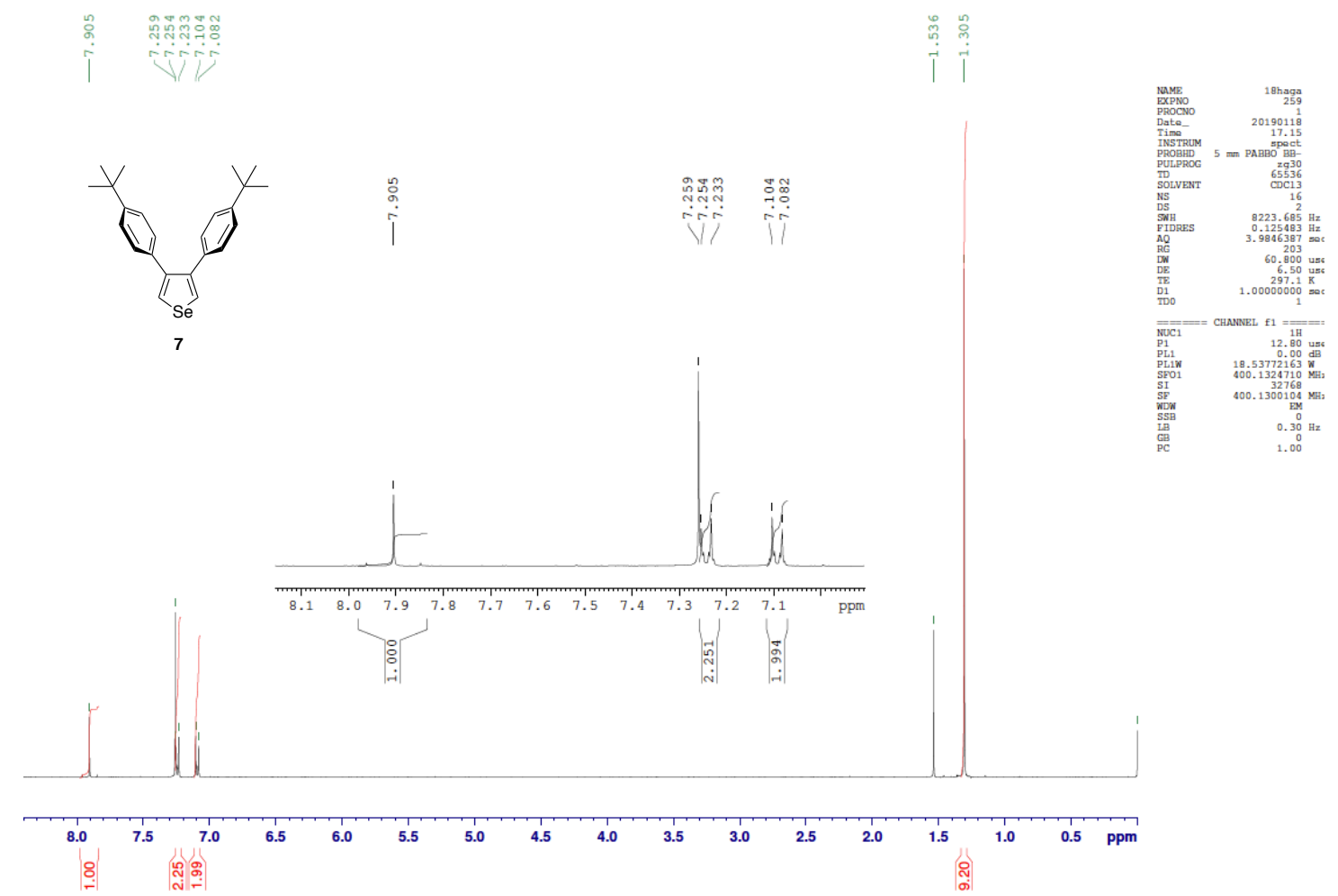


(e) ${ }^{13} \mathrm{C}$ NMR chart of $7\left(100 \mathrm{MHz}, \mathrm{CDCl}_{3}\right)$
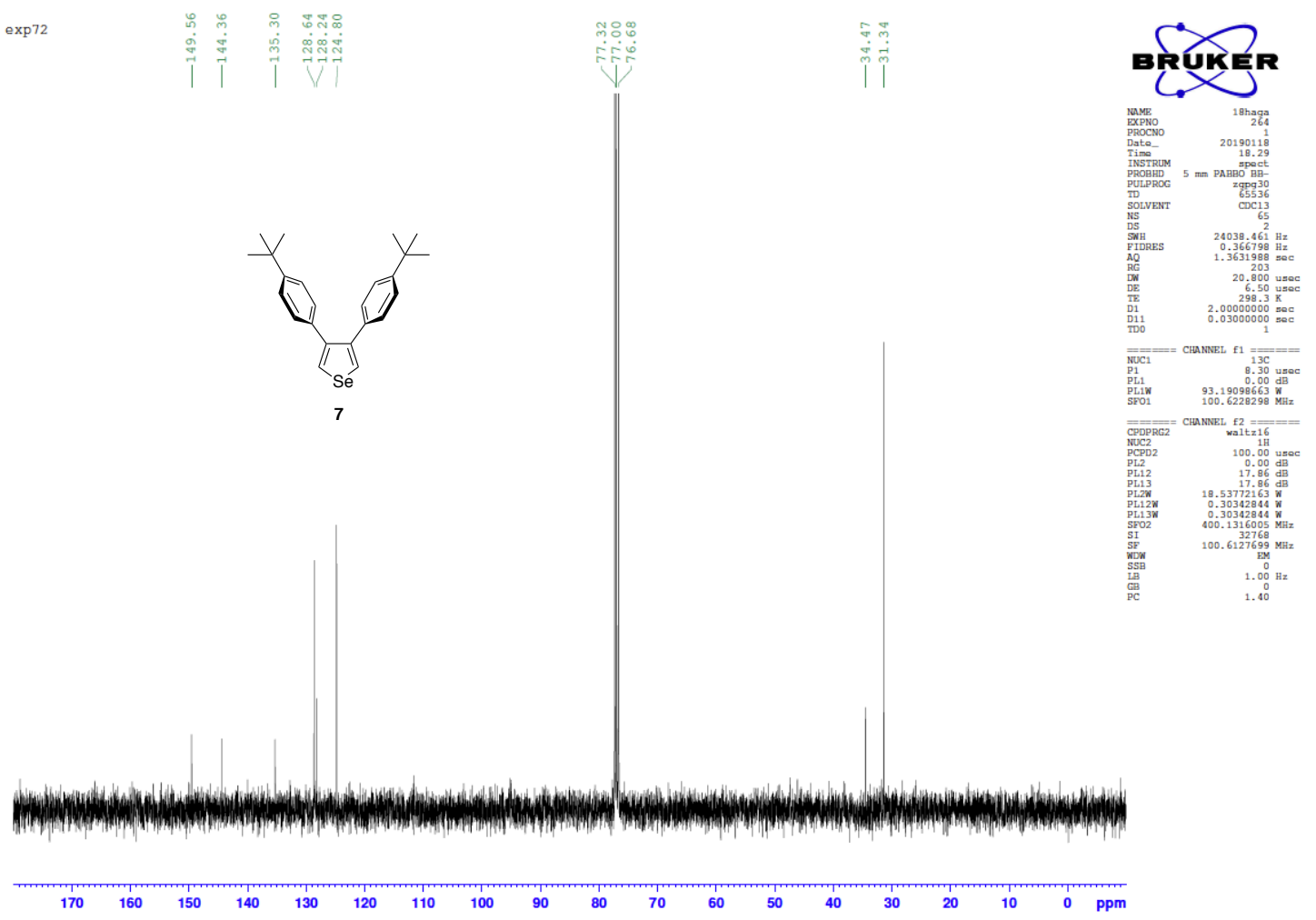

(f) ${ }^{77} \mathrm{Se} \mathrm{NMR}$ chart of $7\left(76 \mathrm{MHz}, \mathrm{CDCl}_{3}\right)$

exp72

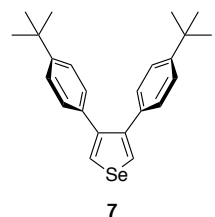

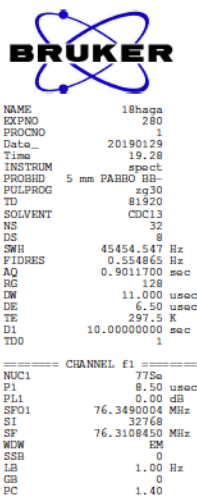

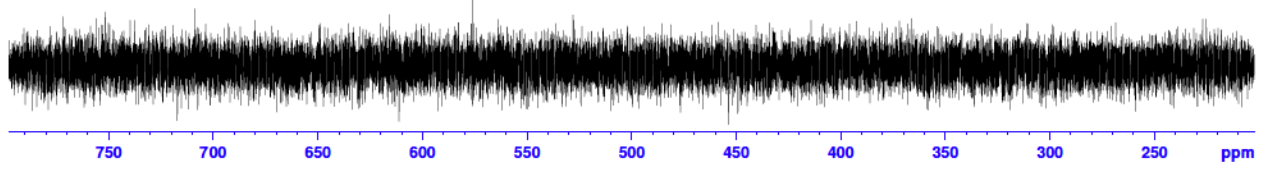


(g) ${ }^{1} \mathrm{H}$ NMR chart of $8\left(400 \mathrm{MHz}, \mathrm{CDCl}_{3}\right)$

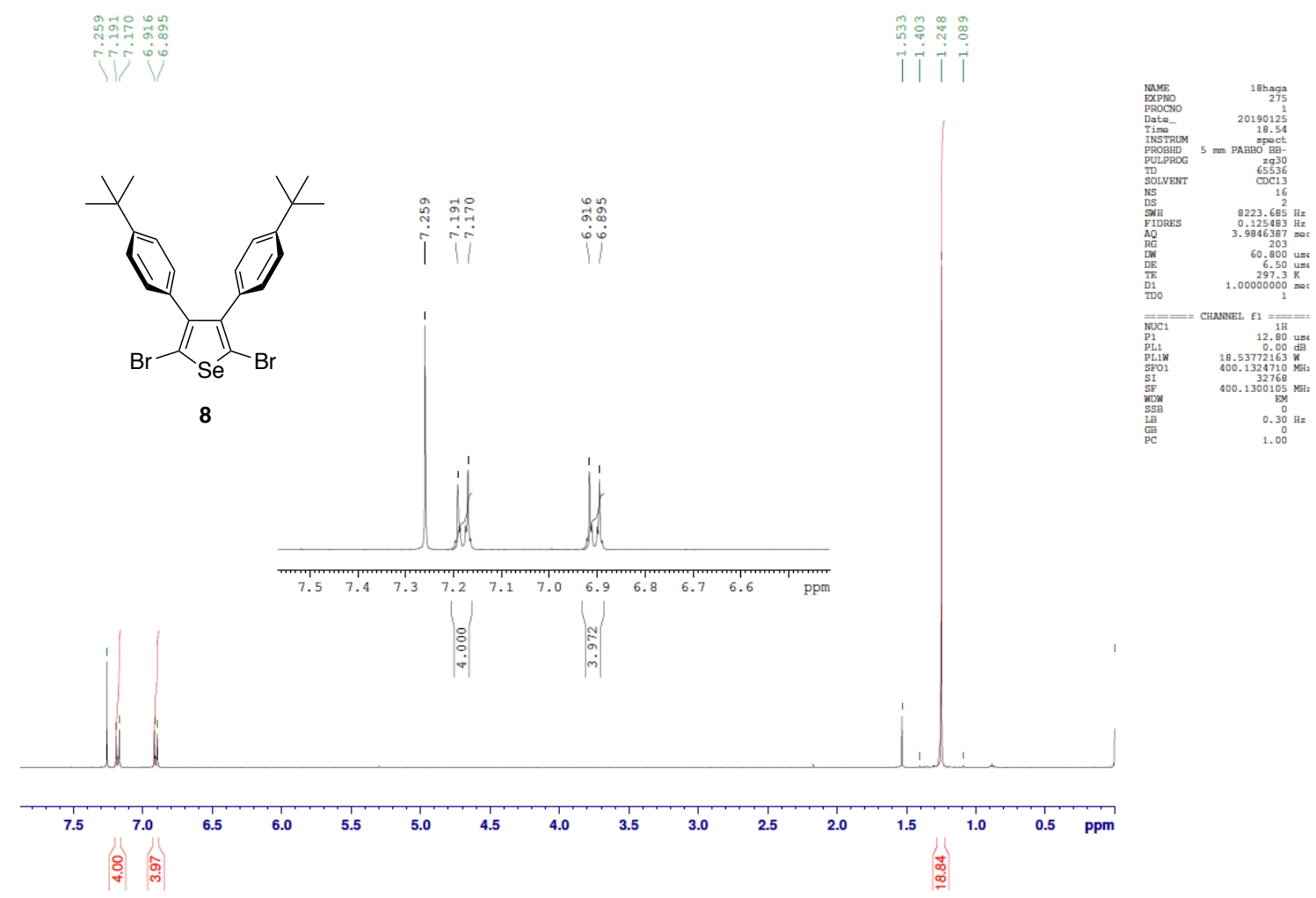

(h) ${ }^{13} \mathrm{C}$ NMR chart of $8\left(100 \mathrm{MHz}, \mathrm{CDCl}_{3}\right)$
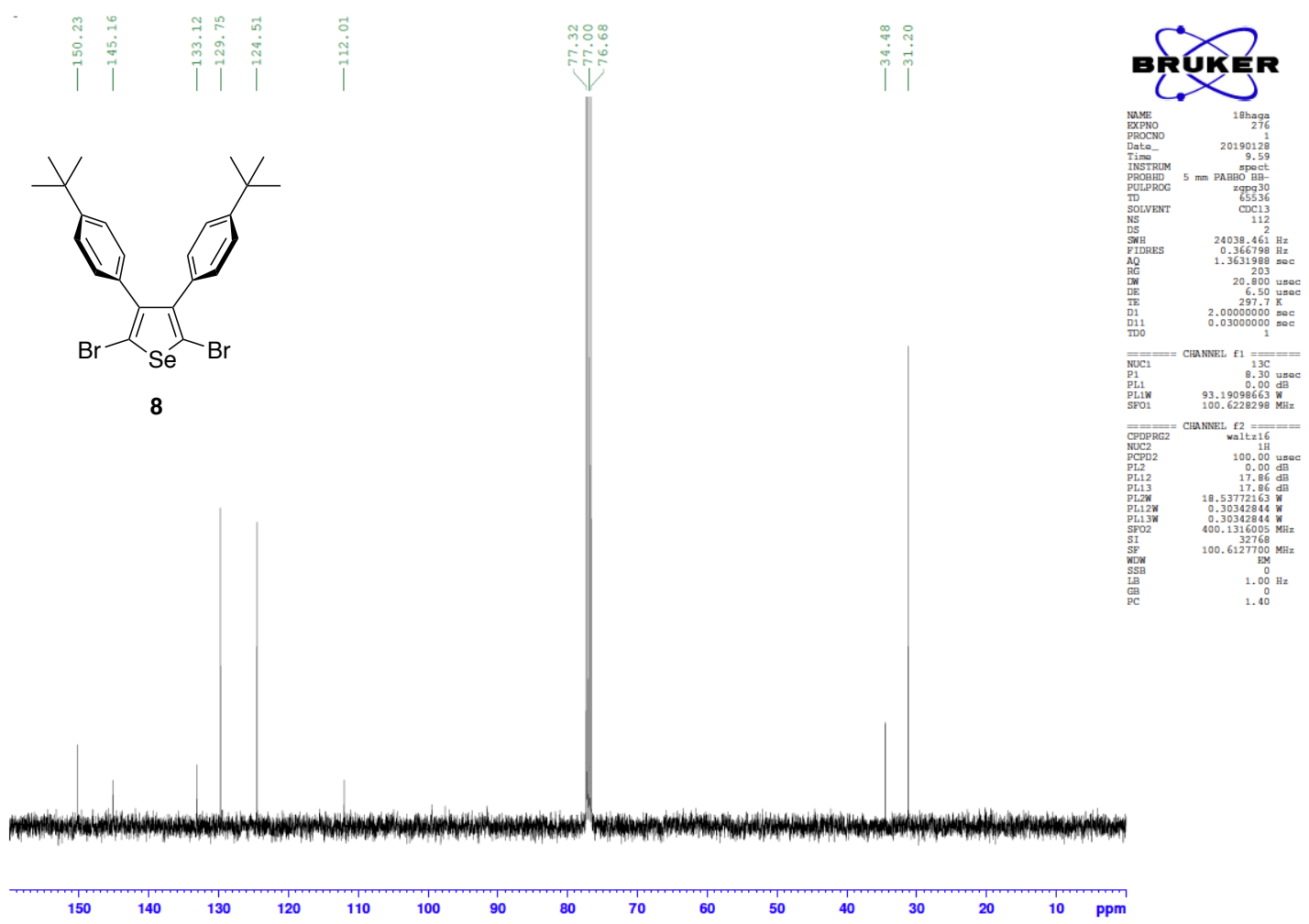
(i) ${ }^{77} \mathrm{Se} \mathrm{NMR}$ chart of $8\left(76 \mathrm{MHz}, \mathrm{CDCl}_{3}\right)$

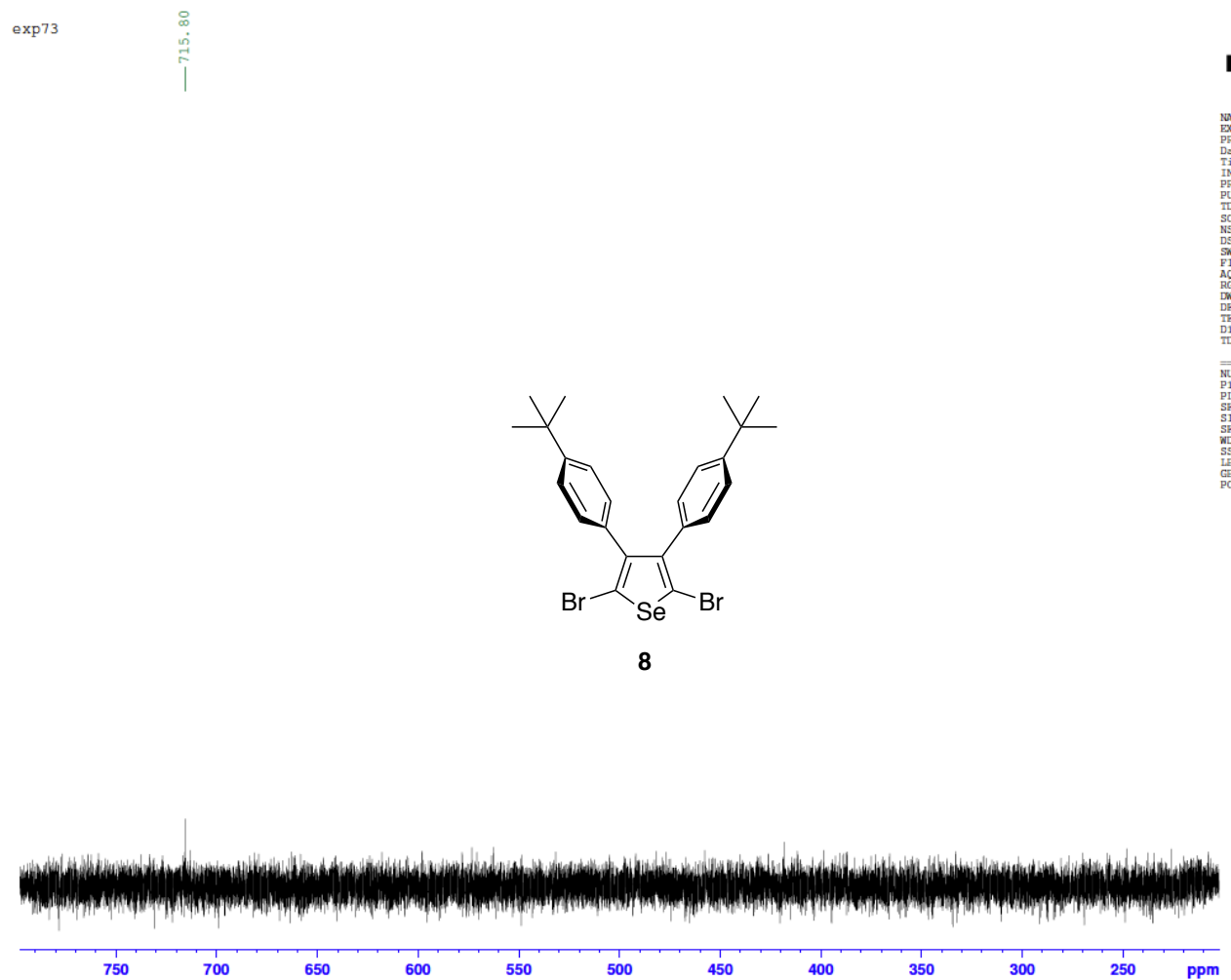


(j) ${ }^{1} \mathrm{H}$ NMR chart of cyclization products after short column (before GPC) (400 MHz, $\mathrm{CDCl}_{3}$ )

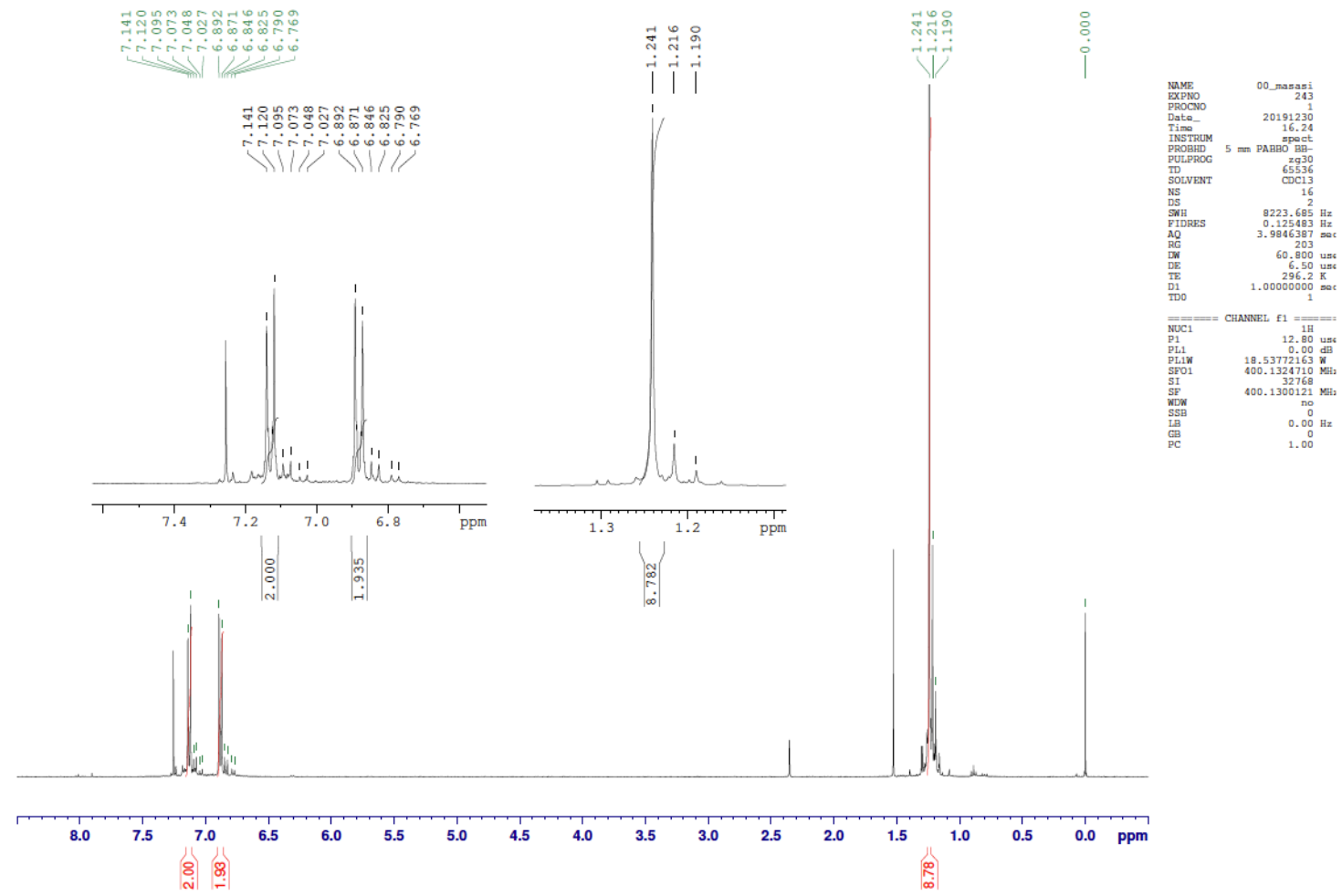

(k) ${ }^{1} \mathrm{H}$ NMR chart of mixture of $\mathbf{4}, \mathbf{5}$, and $\mathbf{6}$ after GPC separation $\left(400 \mathrm{MHz}, \mathrm{CDCl}_{3}\right)$

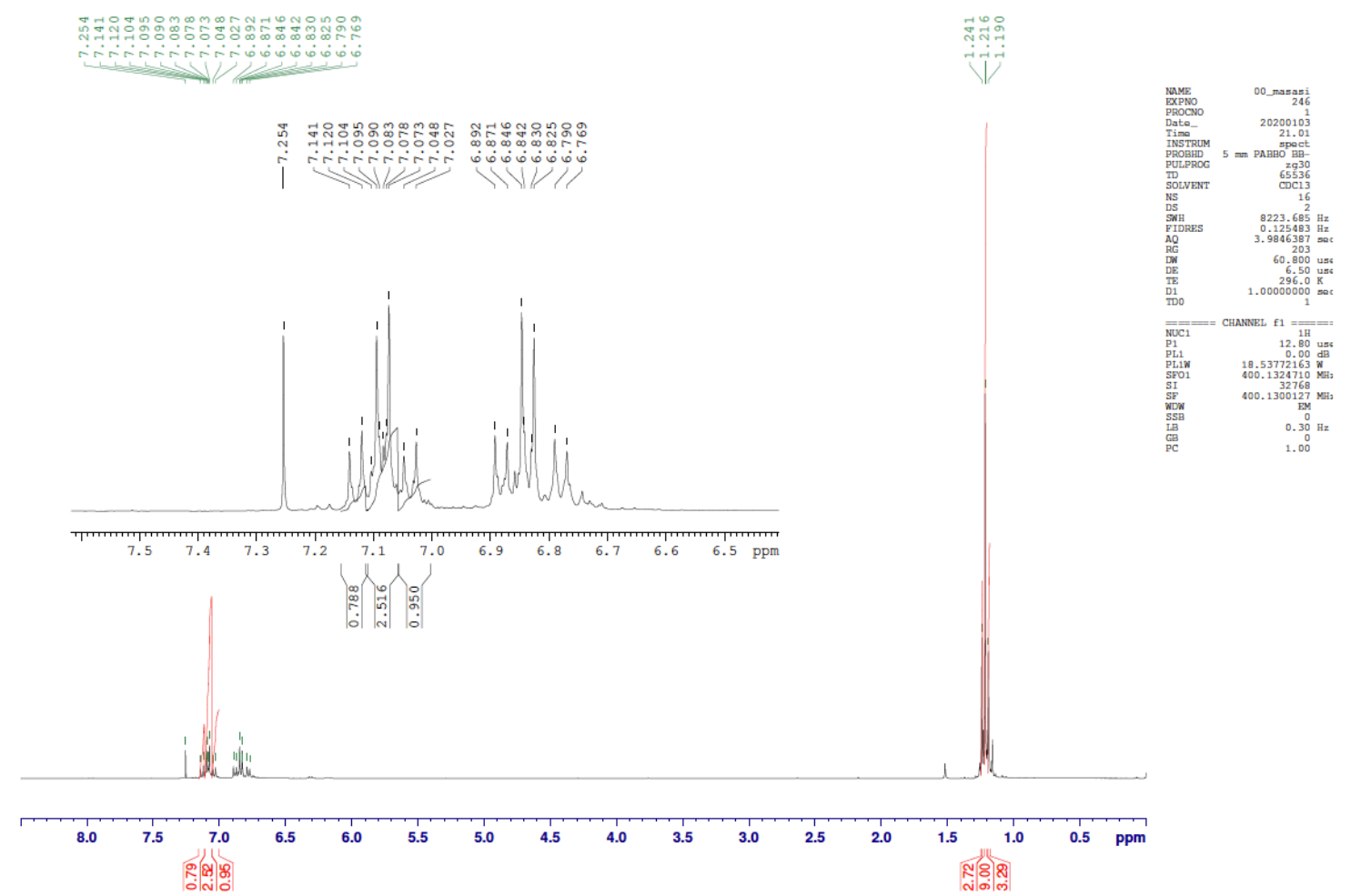


(l) ${ }^{1} \mathrm{H}$ NMR chart of mixture of 5 with small amount of $\mathbf{6}\left(400 \mathrm{MHz}, \mathrm{CDCl}_{3}\right)$

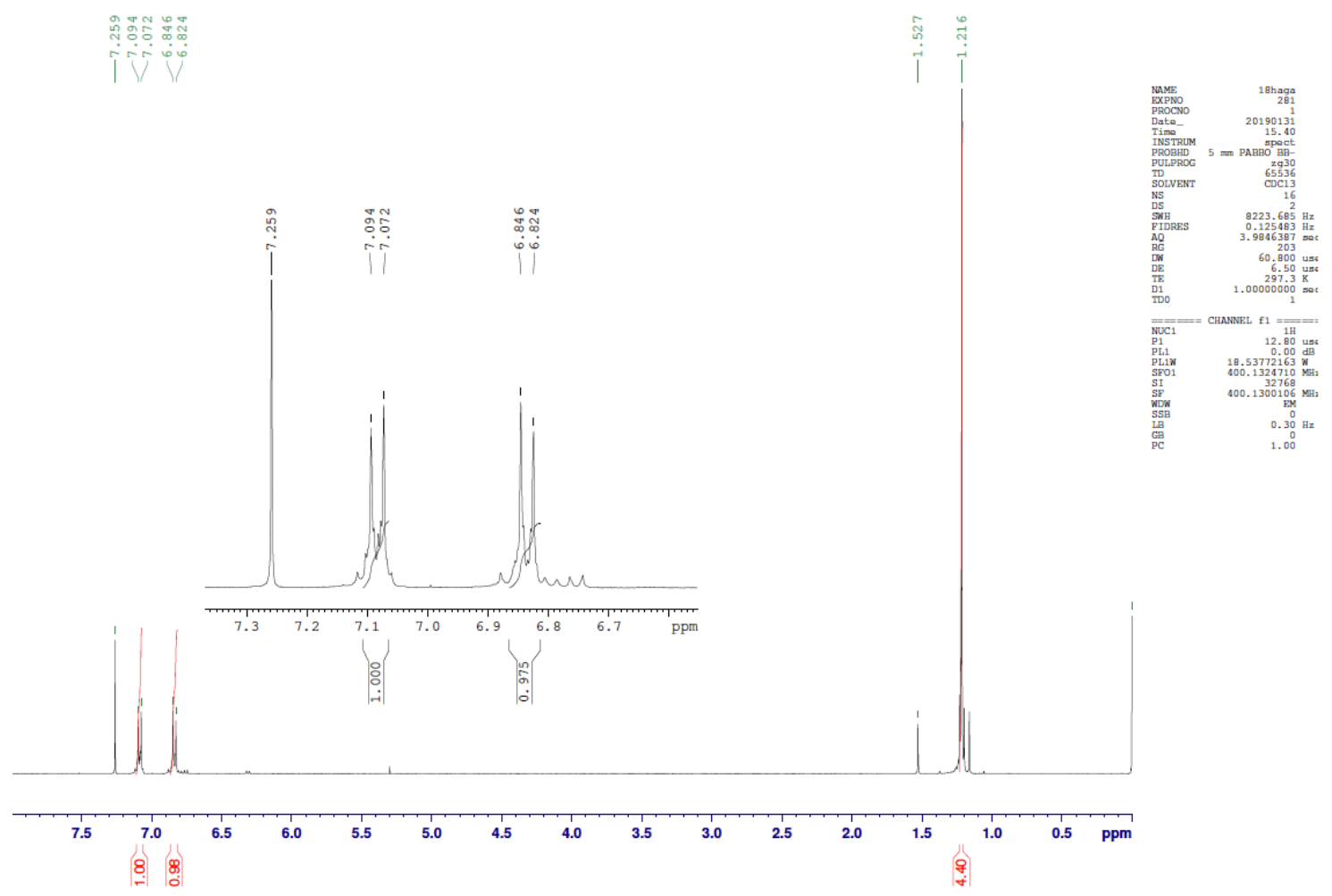

(m) ${ }^{13} \mathrm{C}$ NMR chart of mixture of 5 with small amount of $\mathbf{6}\left(100 \mathrm{MHz}, \mathrm{CDCl}_{3}\right)$

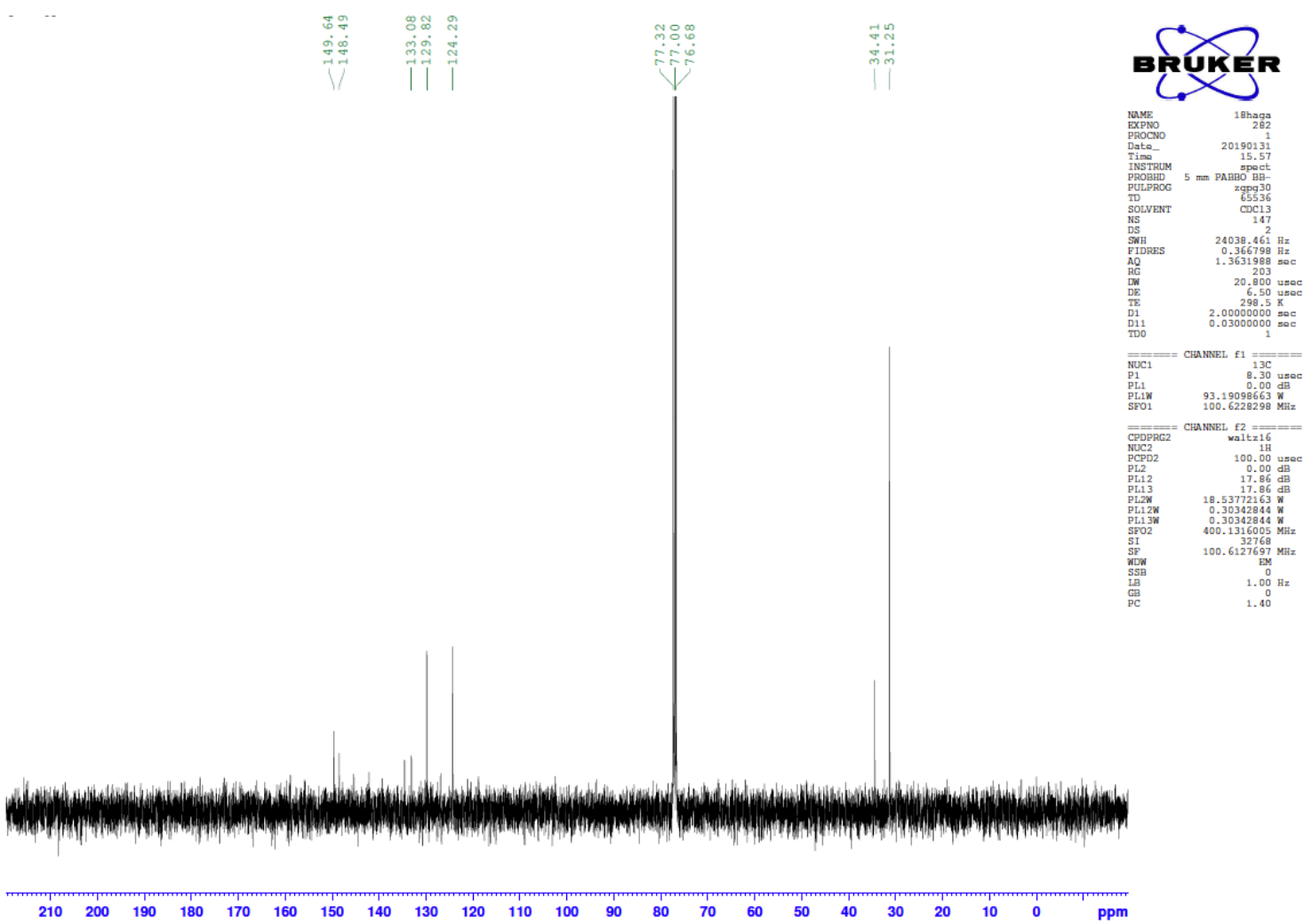


(n) ${ }^{77} \mathrm{Se}$ NMR chart of mixture of 5 with small amount of $6\left(76 \mathrm{MHz}, \mathrm{CDCl}_{3}\right)$
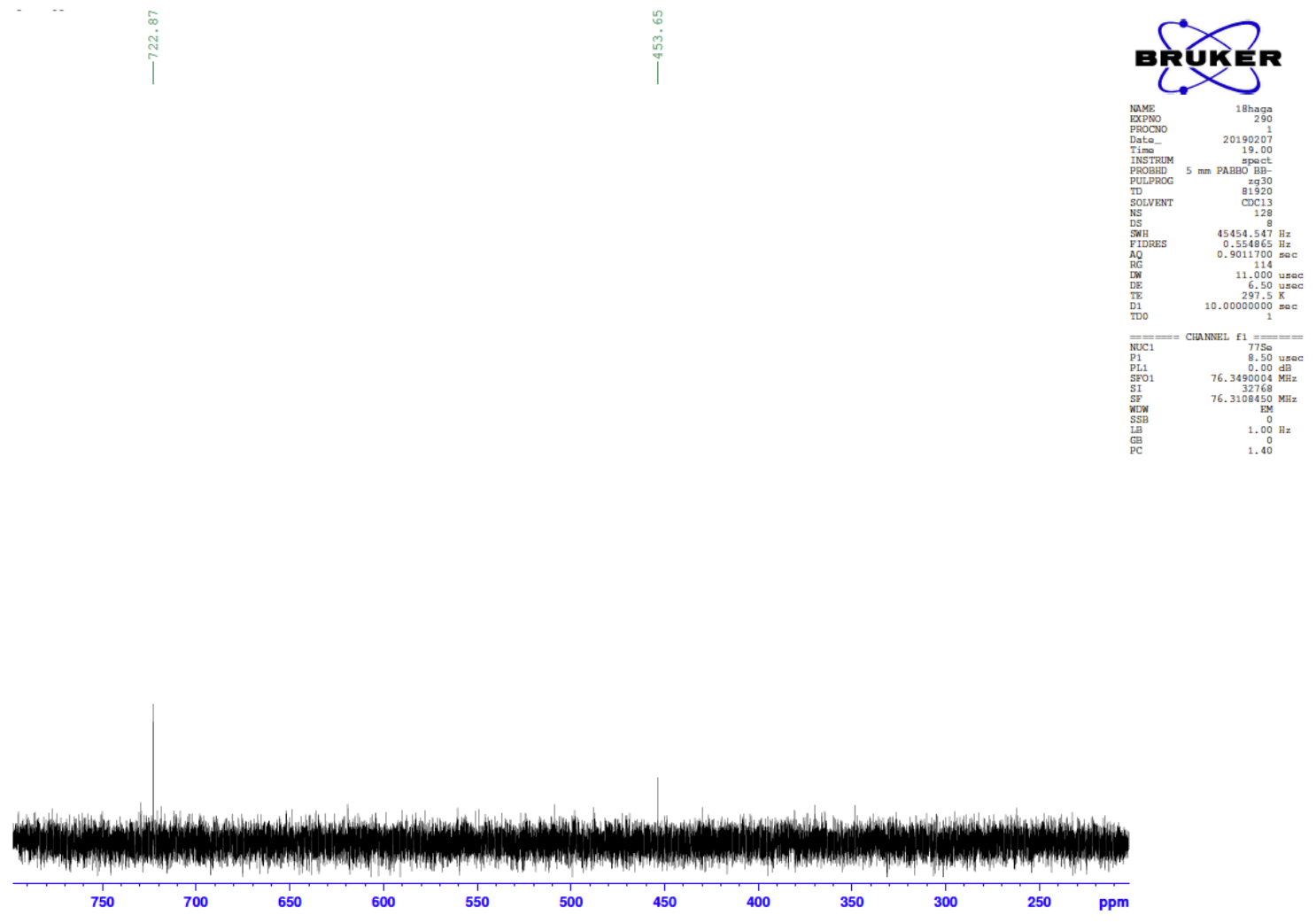


\section{S13. Figure S12. VT-NMR Chart of 4}

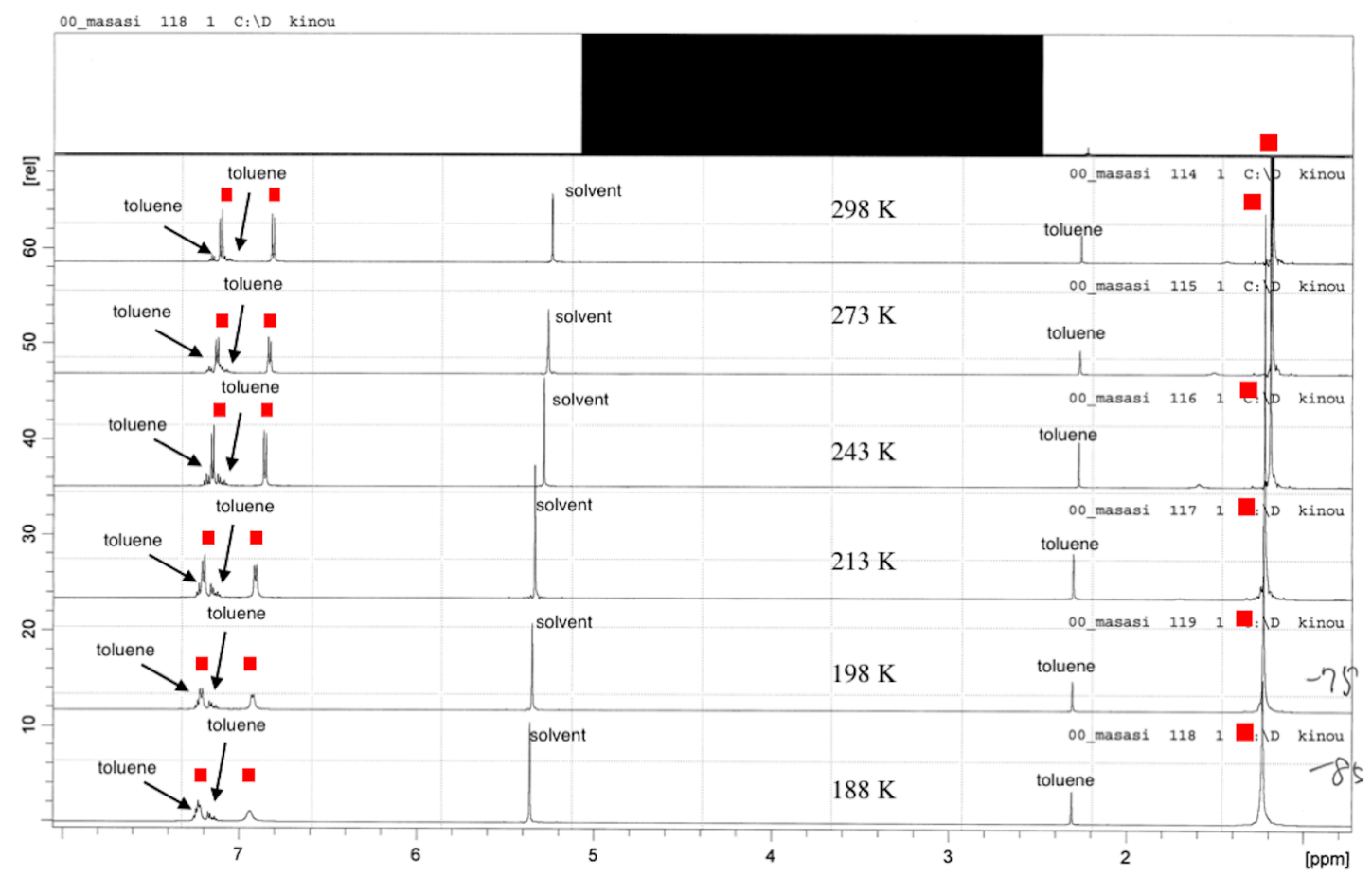

Xerogel of 4 obtained from toluene solution was utilized for the VT-NMR measurements in $\mathrm{CD}_{2} \mathrm{Cl}_{2}$. The VT NMR experiment was carried out at $25^{\circ} \mathrm{C}(298 \mathrm{~K}), 0^{\circ} \mathrm{C}(273 \mathrm{~K}),-30^{\circ} \mathrm{C}(243 \mathrm{~K}),-60^{\circ} \mathrm{C}(213 \mathrm{~K})$, $-75^{\circ} \mathrm{C}(198 \mathrm{~K})$, and $-85^{\circ} \mathrm{C}(188 \mathrm{~K})$. 


\section{S14. Figure S13. MS Spectrum Charts}

(a) ESI-MS of 4

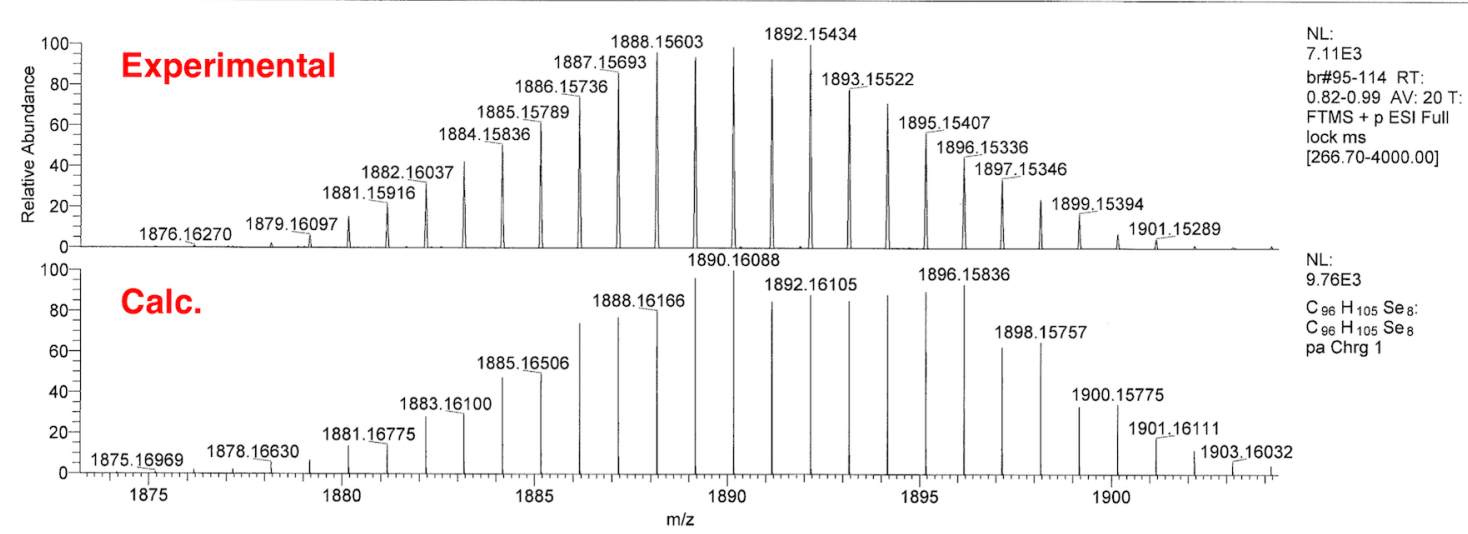

(b) ESI-MS of 5

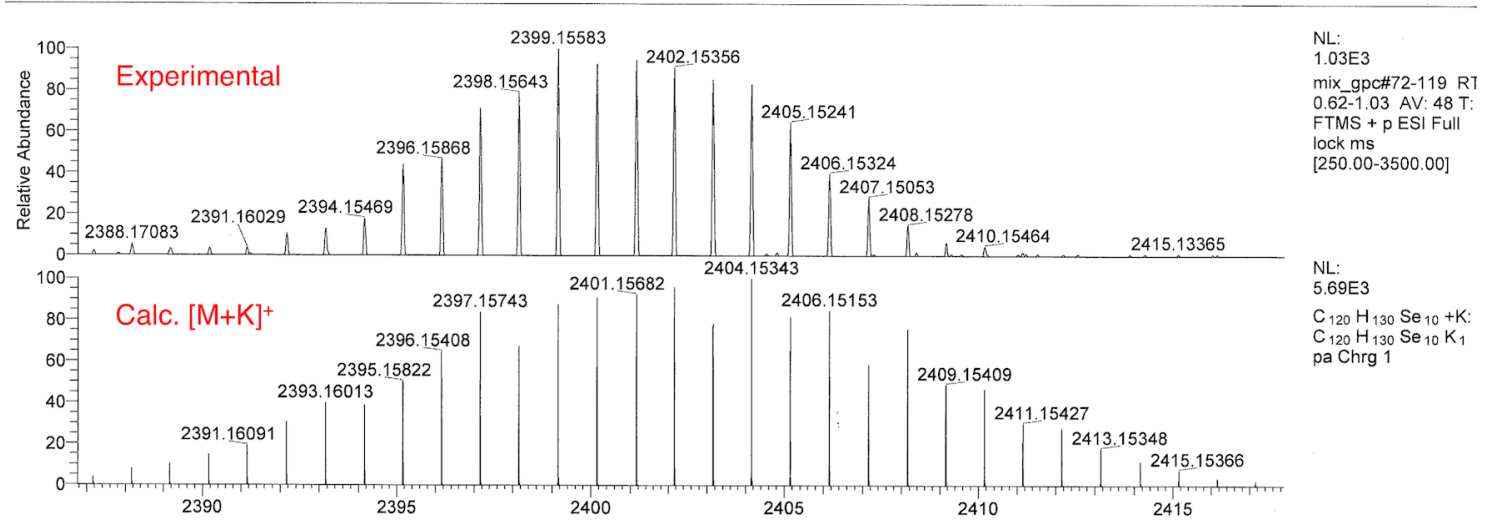

(b) ESI-MS of 6

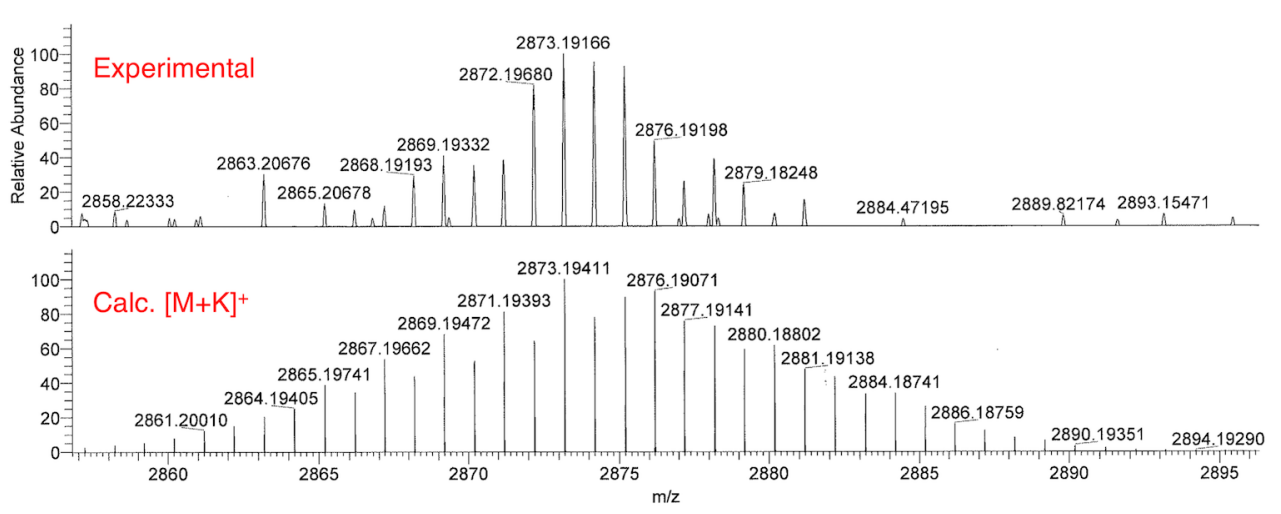

NL:
$2.13 E 2$

mix_gpc\#69-116 RT:

FTMS + p ESI Full

lock ms

${ }_{4.20 E 3}^{N L:}$

$\mathrm{C}_{144} \mathrm{H}_{156} \mathrm{Se}_{12}+\mathrm{K}:$

pa Chrg 1 
(d) ESI-MS of mixture fraction obtained from GPC

\begin{tabular}{l}
\hline mix_gpc \#72-117 RT: $0.62-1.01 \quad$ AV: $46 \quad$ NL: $1.05 E 3$ \\
T: FTMS + p ESI Full lock ms [250.00-3500.00]
\end{tabular}

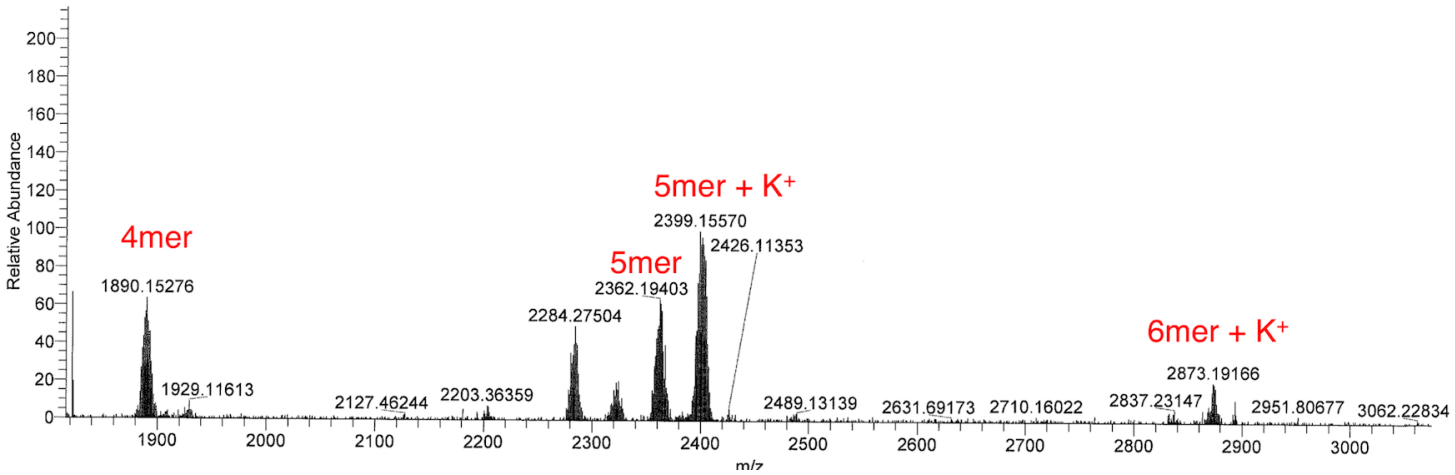




\section{S15. References}

(1) Patra, A.; Wijsboom, Y. H.; Leitus, G.; Bendikov, M., Chem. Mater. 2011, 23, 896-906.

(2) Maeda, H.; Takashima, M.; Sakata, K.; Watanabe, T.; Honda, M.; Segi, M., Tetrahedron Lett. 2011, 52, 415-417.

(3) Amerego, W. L. F.; Chai, C. L. L., Purification of Laboratory Chemicals, Ed. 7, ButterworthHeinemann, Oxford, 2013. 